\title{
Zinc antagonizes iron-regulation of tyrosine hydroxylase activity and dopamine production in Drosophila melanogaster
}

\author{
Guiran Xiao ${ }^{1,2+}$, Mengran Zhao ${ }^{1 \dagger}$, Zhihua $\mathrm{Liu}^{2}$, Fan Du$^{1}$ and Bing Zhou ${ }^{1 *}$ (D)
}

\begin{abstract}
Background: Dopamine (DA) is a neurotransmitter that plays roles in movement, cognition, attention, and reward responses, and deficient DA signaling is associated with the progression of a number of neurological diseases, such as Parkinson's disease. Due to its critical functions, DA expression levels in the brain are tightly controlled, with one important and rate-limiting step in its biosynthetic pathway being catalyzed by tyrosine hydroxylase (TH), an enzyme that uses iron ion $\left(\mathrm{Fe}^{2+}\right)$ as a cofactor. A role for metal ions has additionally been associated with the etiology of Parkinson's disease. However, the way dopamine synthesis is regulated in vivo or whether regulation of metal ion levels is a component of DA synthesis is not fully understood. Here, we analyze the role of Catsup, the Drosophila ortholog of the mammalian zinc transporter SLC39A7 (ZIP7), in regulating dopamine levels.

Results: We found that Catsup is a functional zinc transporter that regulates intracellular zinc distribution between the ER/Golgi and the cytosol. Loss-of-function of Catsup leads to increased DA levels, and we showed that the increased dopamine production is due to a reduction in zinc levels in the cytosol. Zinc ion $\left(\mathrm{Zn}^{2+}\right)$ negatively regulates dopamine synthesis through direct inhibition of $\mathrm{TH}$ activity, by antagonizing $\mathrm{Fe}^{2+}$ binding to $\mathrm{TH}$, thus rendering the enzyme ineffective or non-functional.
\end{abstract}

Conclusions: Our findings uncovered a previously unknown mechanism underlying the control of cellular dopamine expression, with normal levels of dopamine synthesis being maintained through a balance between $\mathrm{Fe}^{2+}$ and $\mathrm{Zn}^{2+}$ ions. The findings also provide support for metal modulation as a possible therapeutic strategy in the treatment of Parkinson's disease and other dopamine-related diseases.

Keywords: Catecholamines up, Zinc, Iron, Tyrosine hydroxylase, Parkinson's disease

\section{Background}

Parkinson's disease (PD) is one of the most common neurodegenerative diseases with clinical manifestation of motor dysfunctions in the form of tremor, rigidity, and bradykinesia. A hallmark of PD is the progressive dopaminergic neuron death mainly in the ventral midbrain substantia nigra pars compacta (SNc) [1]. The exact

\footnotetext{
* Correspondence: zhoubing@mail.tsinghua.edu.cn

${ }^{+}$Guiran Xiao and Mengran Zhao contributed equally to this work.

${ }^{1}$ State Key Laboratory of Membrane Biology, School of Life Sciences, Tsinghua University, Beijing 100084, China

Full list of author information is available at the end of the article
}

molecular cause underlying neural cell death in PD has not been fully elucidated, and no cure is available. It has long been believed that dopamine (DA) deficit, resulted from dopaminergic neuron death, is a key culprit of PD. DA is the key signaling neurotransmitter molecule critical to body movement, cognition, attention, and reward response [2]. Besides PD, abnormal dopaminergic signaling has also been implicated in a number of other diseases, including attention deficit hyperactivity disorder, psychosis, schizophrenia, and depression [3-5]. The entire pathway of DA synthesis has been well established, and the initial and rate-limiting step is catalyzed by the

(c) The Author(s). 2021 Open Access This article is licensed under a Creative Commons Attribution 4.0 International License, which permits use, sharing, adaptation, distribution and reproduction in any medium or format, as long as you give appropriate credit to the original author(s) and the source, provide a link to the Creative Commons licence, and indicate if changes were made. The images or other third party material in this article are included in the article's Creative Commons licence, unless indicated otherwise in a credit line to the material. If material is not included in the article's Creative Commons licence and your intended use is not permitted by statutory regulation or exceeds the permitted use, you will need to obtain permission directly from the copyright holder. To view a copy of this licence, visit http://creativecommons.org/licenses/by/4.0/. The Creative Commons Public Domain Dedication waiver (http://creativecommons.org/publicdomain/zero/1.0/) applies to the data made available in this article, unless otherwise stated in a credit line to the data. 
tyrosine hydroxylase (TH) [6], a metal-dependent enzyme that uses $\mathrm{Fe}^{2+}$ as the cofactor. TH activity is known to be regulated by a complex mechanism including phosphorylation [7]. Since alteration in TH activity and DA synthesis/signaling has been found to be associated with PD and other neurological disorders [8], modulation of $\mathrm{TH}$ activity or DA signaling has been proposed as a potential therapeutic strategy for the treatment of PD $[9,10]$.

There are ample evidences indicative of a role for metal ions in PD etiology [11, 12], consistent with the fact that the quintessential $\mathrm{TH}$ in DA synthesis is a $\mathrm{Fe}^{2+}$-dependent enzyme. Clinic manifestation of manganism, a disorder of manganese toxicity, resembles idiopathic PD as well as other neurodegenerative diseases such as Lou Gehrig's disease and multiple sclerosis [13]. In the dopaminergic neurons of PD patients, the iron level was found to be increased in the substantia nigra but decreased in the globus pallidus [14, 15], and zinc in contrast was abnormally accumulated in the region [15]. Feeding rats with excess zinc was found to trigger degeneration of dopaminergic neurons and decreased DA levels [16, 17], implying abnormally high zinc levels in the dopaminergic neurons of PD patients might be causative. Interestingly, iron content in PD patients' hair was significantly lowered, and zinc content in contrast was increased [18], suggesting a possible alteration and abnormal interplay between these two metal ions in these patients. Whether or not these metal-related defects are part of the underlying cause or aftermath consequences of PD remains to be more affirmatively determined. Furthermore, how these different metal ions might contribute to PD lacks a unified mechanistic understanding.

We have long been interested in understanding how metal ions might contribute to and influence PD and other neurodegenerative diseases. We were particularly interested in a Drosophila mutant named Catecholamines up (Catsup). As the name indicates, in Catsup mutant flies, the DA level was found to be substantially elevated [19]. The corresponding protein Catsup encoded by Catsup/CG10449 has been characterized as a negative regulator of $\mathrm{TH}$ activity during catecholamine biosynthesis [19]. Loss of Catsup function was shown to delay the onset of neurological symptoms, dopaminergic neuron death, and morbidity of flies upon paraquat exposure [20]. Catsup has also been found to participate in the synaptic vesicle loading and release of DA [21], the regulation of tracheal morphogenesis [22], the control of sleep behavior in Drosophila [23], and the maintenance of ER/Golgi function [24]. Interestingly, Catsup is homologous to the mammalian zinc transporter ZIP7 protein (also known as SLC39A7) [25], a member of the ZIP (Zrt/Irt-like protein) family [26]. Mammalian ZIP7 is also known to be localized in the ER and functions in mobilizing zinc from ER into the cytosol [26-29]. It is therefore possible that the Drosophila Catsup might function as a zinc transporter somehow exerting regulatory control of DA synthesis, and this likely scenario could explain how zinc may contribute to dopaminergic neural degeneration.

In the current study, we report both genetic and biochemical studies demonstrating that Catsup is indeed a functional zinc transporter participating in zinc homeostasis in D. melanogaster. Catsup knockdown leads to zinc reduction in the cytosol. More importantly, we demonstrated that cytosolic zinc reduction triggered $\mathrm{TH}$ activation and DA increase. We further showed that zinc affects $\mathrm{TH}$ activity thus DA synthesis by antagonizing $\mathrm{Fe}^{2+}$, the indispensable cofactor of TH. Our study has uncovered a previously unknown mechanism underlying the regulation of DA synthesis. The finding provides a mechanistic framework to understand how zinc and iron ions could affect DA synthesis therefore contribute to PD. Our finding also points to the utility of zinc limitation as a possible novel therapeutic strategy in the treatment of PD and implies a role of metal homeostasis in the regulation of mood or behavior of animals.

\section{Results}

\section{Catsup is a zinc transporter functionally analogous to human ZIP7}

There are at least ten putative ZIPs in the D. melanogaster genome [30-33]. Among them, Catsup/CG10449 shares the highest overall homology with human ZIP7 (hZIP7, also known as SLC39A7, 52\% identity and 64\% similarity), which functions as a zinc transporter located on the ER/Golgi [26]. Under normal dietary conditions, ubiquitous RNAi of Catsup by daughterless Da-Gal4 produced developmental arrest at the embryonic stage (0\% eclosion rate) (Fig. 1A). This eclosion defect resulting from Catsup knockdown could be effectively rescued by hZIP7 from $\sim 0$ to $\sim 83 \%$ (Fig. 1A). As a control, expression of dZIP13/CG7816 (also named Zip99C), a close homolog of hZIP7 but previously shown as an iron transporter located on the ER/Golgi [30], had no rescuing effect on Catsup RNAi (Fig. 1A). When Catsup was knocked down in the central nervous system (CNS), directed by the pan-neuronal Elav-Gal4 [34], the flies exhibited early adult death or much reduced lifespan at $25^{\circ} \mathrm{C}$ (Fig. 1B). The effects of hZIP7 and dZIP13 overexpression (OE) on the lifespan of Catsup RNAi were also analyzed. hZIP7 exhibited a strong rescuing effect, from $\sim 8$ to $\sim 32$ days, on the medium lifespans of Catsup RNAi flies raised at $25^{\circ} \mathrm{C}$, whereas dZIP13 OE had no effect (Fig. 1B). When Catsup was knocked down with Elav-Gal4, blackspots (cell death) appeared in the eyes 

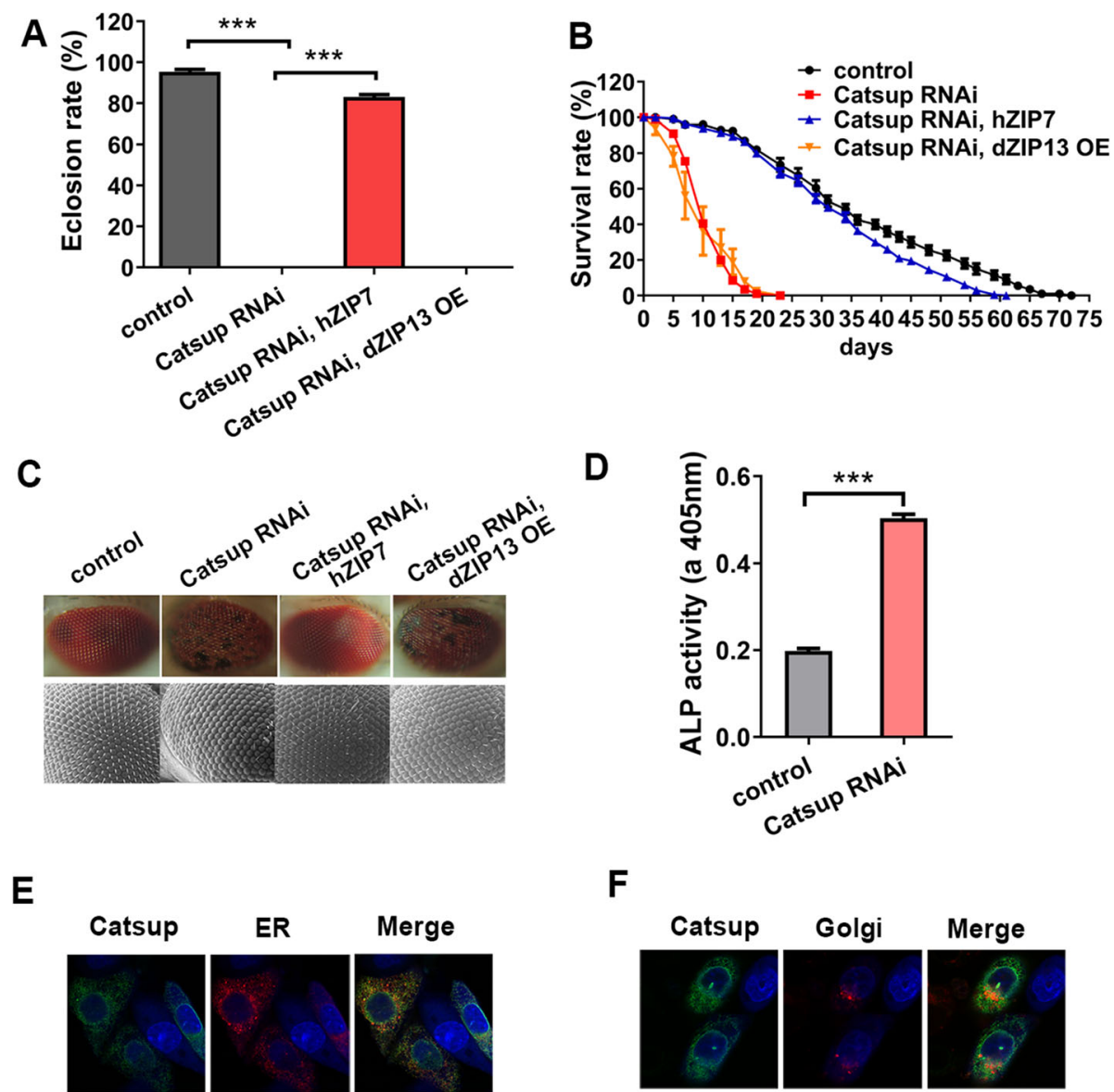

Fig. 1 Catsup is the Drosophila ortholog of hZIP7 and functions as a zinc transporter. A The eclosion defect from ubiquitous Catsup RNA interference (Da-Gal4>Catsup RNAi) could be effectively rescued by hZIP7 expression, but not by dZIP13 OE. Genotypes of the flies used were DaGal4> w ${ }^{1118}$ (control); Da-Gal4>Catsup RNAi; Da-Gal4>Catsup RNAi, hZIP7; Da-Gal4>Catsup RNAi, dZIP13 OE. All values are presented as mean \pm $\mathrm{SEM} ; n=6 .{ }^{* * *} p<0.001$. B The shortened lifespan of Catsup RNAi in the central nervous system was rescued by $h Z I P 7$, but not $d Z I P 13$ OE. Genotypes of the flies used were Elav-Gal4> $w^{1118}$ (control); Elav-Gal4>Catsup RNAi; Elav-Gal4>Catsup RNAi, hZIP7; Elav-Gal4>Catsup RNAi, dZIP13 OE. $n=6 . C h Z I P 7$, but not dZIP13 OE, restored the eye blackspots of Catsup RNAi flies. Genotypes of the flies used were Elav-Gal4> $w^{1118}$ (control); Elav-Gal4>Catsup RNAi; Elav-Gal4>Catsup RNAi, hZIP7; Elav-Gal4>Catsup RNAi, dZIP13 OE. $n \geq 6$. D The ALP activity, indicated by absorbance at 405 $\mathrm{nm}$, was increased in the heads of Catsup RNAi flies when driven by the pan-neuronal driver Elav-Gal4, suggesting that Catsup RNAi in neurons led to zinc elevation in the Golgi. Genotypes of the flies used were Elav-Gal4> $w^{1718}$ (control) and Elav-Gal4>Catsup RNAi. E, F The localization of Catsup in Chinese hamster ovary (CHOK1) cells. Catsup-FLAG was detected by anti-FLAG antibody. This immunofluorescence staining showed that Catsup partially co-localizes with the ER/Golgi in CHOK1 cells

(Fig. 1C). Consistent with the results described above, the blackspots were also markedly rescued by hZIP7 but not dZIP13 OE (Fig. 1C). The secretory enzyme alkaline phosphatase (ALP) activity is dependent on zinc loading in the Golgi, so its activity is responsive to zinc [35, 36]. In order to test whether zinc level was affected by Catsup in neurons, we tested the ALP activity of Catsup RNAi flies driven by Elav-Gal4 (Fig. 1D). The results indicated that Catsup RNAi in neurons led to zinc elevation in the Golgi.

To examine the intracellular position of Catsup, a Cterminal flag-tagged Catsup was introduced into the Chinese hamster ovary (CHO-K1) cells. Immunofluorescence staining indicated that Catsup partially co-localizes with the ER/Golgi markers in CHO-K1 cells (Fig. 1E, F), consistent with the reported mammalian ZIP7 subcellular location [26]. Previous fluorescence imaging with the zinc indicator Zinpyr-1 has shown decreased zinc accumulation in the Golgi compartment in CHO-K1 cells expressing Catsup [37]. Based on the above results, that is, sequence homology, functional substitution, and subcellular localization, as well as roles in subcellular zinc distribution, it is reasonable to conclude that Catsup, like hZIP7, is a zinc transporter that mediates the transport of zinc from the ER/Golgi apparatus to the cytoplasm of the cell. 
Catsup loss results in detrimental organellar zinc accumulation and cytosolic zinc reduction

Catsup functions as a transporter that redistributes zinc from the ER/Golgi to the cytosol. While Catsup reduction is associated with increased DA production, more severe loss of Catsup activity is lethal to the larvae [14]. In fact, ubiquitous Catsup knockdown produced no adult flies (Fig. 1B), and strong loss-of-function Castup mutant alleles have to be maintained in the heterozygous state with the wild-type allele [19]. This lethality could arise, directly or indirectly, from zinc dyshomeostasis either from zinc accumulation in the ER/Golgi and other organelles where Catsup is likely located, or zinc deficiency in the cytosol. In order to distinguish these two possibilities, we checked whether the phenotypes of Catsup RNAi could be rescued or aggravated by other zinc transporters. Catsup was specifically knocked down in the dopaminergic cells with the TH-Gal4 driver [38]. Approximately $63 \%$ of these Catsup RNAi flies were able to eclose compared to the control (near 100\%), and their lethality could be rescued by hZIP7 (Fig. 2A). If Castup RNAi lethality had been due to cytosolic zinc reduction, we expected that overexpression of $d Z n T 1 / C G 17723$ (also named ZnT63C) [39, 40], a well-established plasma membrane zinc transporter, would exacerbate the toxicity of Catsup knockdown. Contrary to this notion, $d Z n T 1$ OE, which should reduce intracellular zinc level, in fact alleviated the lethality of Catsup RNAi (Fig. 2A). As controls, the eclosion of $d Z n T 1$ OE or hZIP7 driven by TH-Gal4 showed no defects (Fig. 2A). This suggests that the death from Catsup RNAi came from zinc toxicity, i.e., compartmental zinc accumulation or associated processes such as trafficking in the secretory pathway.
Indeed, ER stress has been well noted in previous studies after hZIP7 loss [24, 41].

The sensitivity to zinc of Catsup knockdown (THGal4>Catsup RNAi) was further confirmed with a dietary zinc supplement. Supplementation of additional $\mathrm{ZnCl}_{2}$ significantly worsened the survival defect from 64 to $39 \%$ (Fig. 2B).

\section{TH activity enhancement in Catsup mutants arises from} cytosolic zinc reduction instead of organelle zinc stress Catsup is responsible for intracellular zinc redistribution between the cytosol and the ER/Golgi. As mentioned, the disturbance of this distribution causes lethality as well as elevated DA production. Zinc accumulation in the secretory compartments is the basis for lethality. But what causes the DA increase? Is the observed elevated $\mathrm{TH}$ activity due to zinc elevation in the secretory compartments, or zinc reduction in the cytosol, or both? We first confirmed that $\mathrm{TH}$ activity is indeed modulated by redistributing intracellular zinc. This is meaningful since it could be argued that Catsup might be associated with some unidentified novel property other than zinc transport. dZnT7 is a well-characterized ER/Golgi-resident zinc transporter which functions in the opposite direction to Catsup [42]. Catsup RNAi and $d Z n T 7$ OE both reduce cytosolic zinc and increase ER/Golgi zinc levels. When driven with the dopaminergic neuron driver $\mathrm{TH}$ Gal4, Catsup RNAi and $d Z n T 7$ OE exhibited respectively $\sim 300 \%$ and $200 \%$ more $\mathrm{TH}$ activity in the head, as compared to the control (Fig. 3A). Consistently, DA levels in the Catsup RNAi and $d Z n T 7$ OE fly heads increased nearly $100 \%$ as compared to the control (Fig. 3B). This further strengthens the conclusion that increased $\mathrm{TH}$
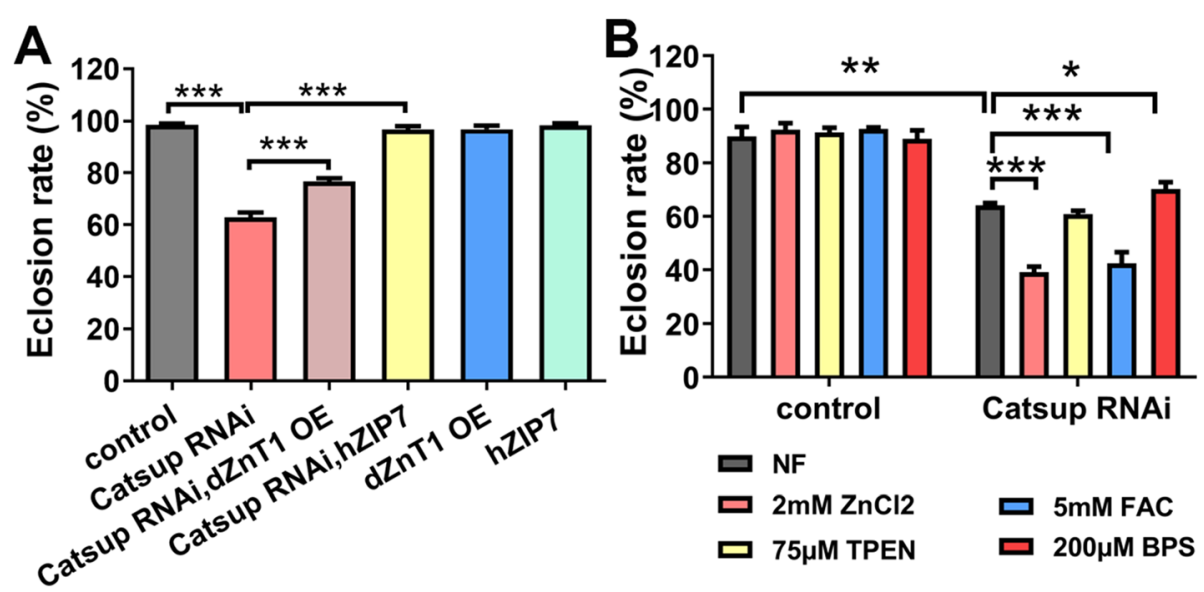

Fig. 2 Catsup knockdown in dopaminergic neurons results in not only zinc but also iron sensitivities due to compartmentalized zinc toxicity. A The decreased eclosion defect of Catsup RNAi flies could be significantly rescued by hZIP7 or dZnT1 OE. Genotypes of the flies used were THGal4> w ${ }^{1118}$ (control); TH-Gal4>Catsup RNAi; TH-Gal4>Catsup RNAi, dZnT1 OE; TH-Gal4>Catsup RNAi, hZIP7. All values are presented as mean \pm SEM; $n=6$. ${ }^{* *} p<0.001$. B The decreased eclosion defect of Catsup RNAi flies could be significantly influenced by dietary zinc or iron. The eclosion defect became deteriorated after $\mathrm{ZnCl}_{2}$ and FAC supplementation. Genotypes of the flies used were $\mathrm{TH}-\mathrm{Gal} 4>\mathrm{w}^{1118}$ (control) and $\mathrm{TH}-\mathrm{Gal} / 4>\mathrm{Cats}$ up RNAi. All values are presented as mean $\pm \mathrm{SEM} ; n=6 .{ }^{*} p<0.05,{ }^{* *} p<0.01,{ }^{* * *} p<0.001$ 

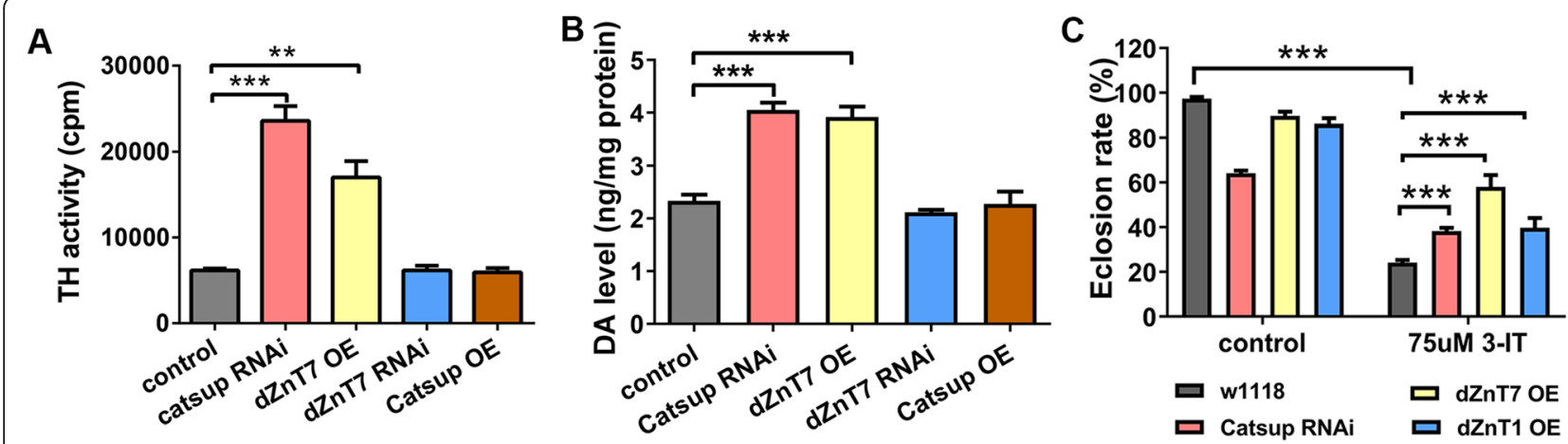

Fig. 3 TH activity elevation in Catsup RNAi is due to cytosolic zinc reduction. A Either Catsup RNAi or dZnT7 OE in the dopaminergic neurons significantly elevated TH activity. Genotypes of the flies used were TH-Gal4>w ${ }^{1118}$ (control), TH-Gal4>Catsup RNAi, TH-Gal4>dZnT7 OE, THGal4>dZnT7 RNAi, and TH-Gal4>Catsup OE. All values are presented as mean \pm SEM and included in Additional file 2 : table $\mathrm{S1} ; \mathrm{n}=4$. ${ }^{* *} p<0.01$, ${ }^{* * *} p<0.001$. B DA level was elevated in Catsup RNAi and dZnT7 OE fly heads. Genotypes of the flies used were TH-Gal4>W ${ }^{1118}$ (control), THGal4>Catsup RNAi, TH-Gal4>dZnT7 OE, TH-Gal4>dZnT7 RNAi, and TH-Gal4>Catsup OE. All values are presented as mean \pm SEM and included in Additional file 2: table S2; $n \geq 3$. ${ }^{* *} p<0.001$. C Expression of zinc exporters enabled resistance to 3-IT, a dopamine synthesis inhibiter. Genotypes of the flies used were TH-Gal4> $w^{1718}$ (control), TH-Gal4>Catsup RNAi, TH-Gal4>dZnT7 OE, and TH-Gal4>dZnT1 OE. All values are presented as mean $\pm \mathrm{SEM} ; n=6 .{ }^{*} p<0.05,{ }^{* *} p<0.01,{ }^{* * *} p<0.001$

activity in Catsup mutant flies is due to zinc dyshomeostasis, instead of some possible novel property other than zinc transport associated with dZIP7 (Catsup).

We then asked whether the zinc accumulation in the secretory compartments or the accompanied cytosolic zinc reduction would enhance DA synthesis. It is known that the lack of DA would lead to death in the flies. Indeed, when the fly was fed with the TH inhibitor 3iodotyrosine (3-IT), the endogenous DA pool was significantly depleted [20] and the viability strongly suppressed (Fig. 3C). As expected, Catsup RNAi and $d Z n T 7$ OE significantly rescued the viability (Fig. 3C). If the effect of Catsup on $\mathrm{TH}$ is through cytosolic zinc reduction, we would expect that the expression of the plasma membrane-resident zinc exporter dZnT1 also confers a rescue. Consistent with this notion, dZnT1 OE in the brain enabled more survivals under 3-IT treatment (Fig. 3C). This argues against the model that zinc accumulation in the ER/Golgi enhanced TH activity.

\section{Catsup loss may affect TH activity independent of TH phosphorylation and $\mathrm{BH}_{4}$ synthesis}

Protein phosphorylation is one of the major mechanisms so far reported regulating TH activity [43]. Drosophila $\mathrm{TH}$ is encoded by the gene named pale [44, 45]. Bioinformatics analysis showed that Drosophila TH is similar to human $\mathrm{TH}[44,46]$, sharing $54 \%$ identity and $74 \%$ similarity (Fig. 4A). The lack of commercial antibodies able to recognize phosphorylated Drosophila TH, so we tested in human neuroblastoma cells (SH-SY5Y cells) the effect of metals on mammalian $\mathrm{TH}$ phosphorylation at Ser40, the major player on $\mathrm{TH}$ activity among the three phosphorylation sites and the only one conserved between the fly and mammals [43, 47]. Western analysis indicated that the phosphorylation of $\mathrm{TH}$ at Ser40 was not prominently and consistently affected by zinc, iron, and chelators (Fig. 4B, Additional file 1: Figure S1 and Additional file 1: Figure S6). In other words, the regulatory effect of zinc or iron on $\mathrm{TH}$ activity may not be attributed to changes in phosphorylation.

$\mathrm{TH}$ requires tetrahydrobiopterin $\left(\mathrm{BH}_{4}\right)$ as a cofactor for its activity [48]. Catsup mutations resulted in strongly elevated $\mathrm{BH}_{4}$ levels [21]. So, we wondered if the effect of zinc on $\mathrm{TH}$ activity is via its influence on $\mathrm{BH}_{4}$. The enzyme GTP cyclohydrolase I (GTPCH) is the rate-limiting enzyme for $\mathrm{BH}_{4}$ biosynthesis [21]. In Drosophila, GTP cyclohydrolase I (GTPCH) is encoded by Punch (Pu) [49]. Ubiquitous RNAi of $P u$ by $\mathrm{Da}$-Gal4 led to eclosion defects in Drosophila, which could be rescued by supplement of 6, 7-dimethyl 5, 6, 7, 8-tetrahydropterin $\left(\mathrm{DMPH}_{4}\right)$, a synthetic cofactor for $\mathrm{TH}$ similar to but more stable than the natural cofactor (6R)-5,6,7,8-tetrahydrobiopterin $\left(\mathrm{BH}_{4}\right)$ (Fig. 4C) $[19,50]$. This indicates that dietary $\mathrm{DMPH}_{4}$ is physiologically accessible to $\mathrm{TH}$ in vivo. Consistently, it is also reported that pre-feeding flies with $\mathrm{BH}_{4}(0.34 \mathrm{mg} / \mathrm{mL})$ could inhibit the firing of the NPF neurons [51]. Nevertheless, for normal flies reared on normal diet, we found that $\mathrm{TH}$ activity remained unaffected with additional $\mathrm{BH}_{4}$ supplementation (Fig. 4D). We consider this negative finding supporting the hypothesis that the amount of endogenous $\mathrm{BH}_{4}$ is probably saturated for the activation of $\mathrm{TH}$ under normal conditions, and additional $\mathrm{BH}_{4}$ may not further improve the $\mathrm{TH}$ enzyme activity. In the case of Catsup mutation, although $\mathrm{TH}$ activity is elevated, TH protein level is not [19]. This suggests that the observed increase in $\mathrm{TH}$ activity in Catsup loss flies may at least partially come from another route different from the increased $\mathrm{BH}_{4}$ synthesis. 

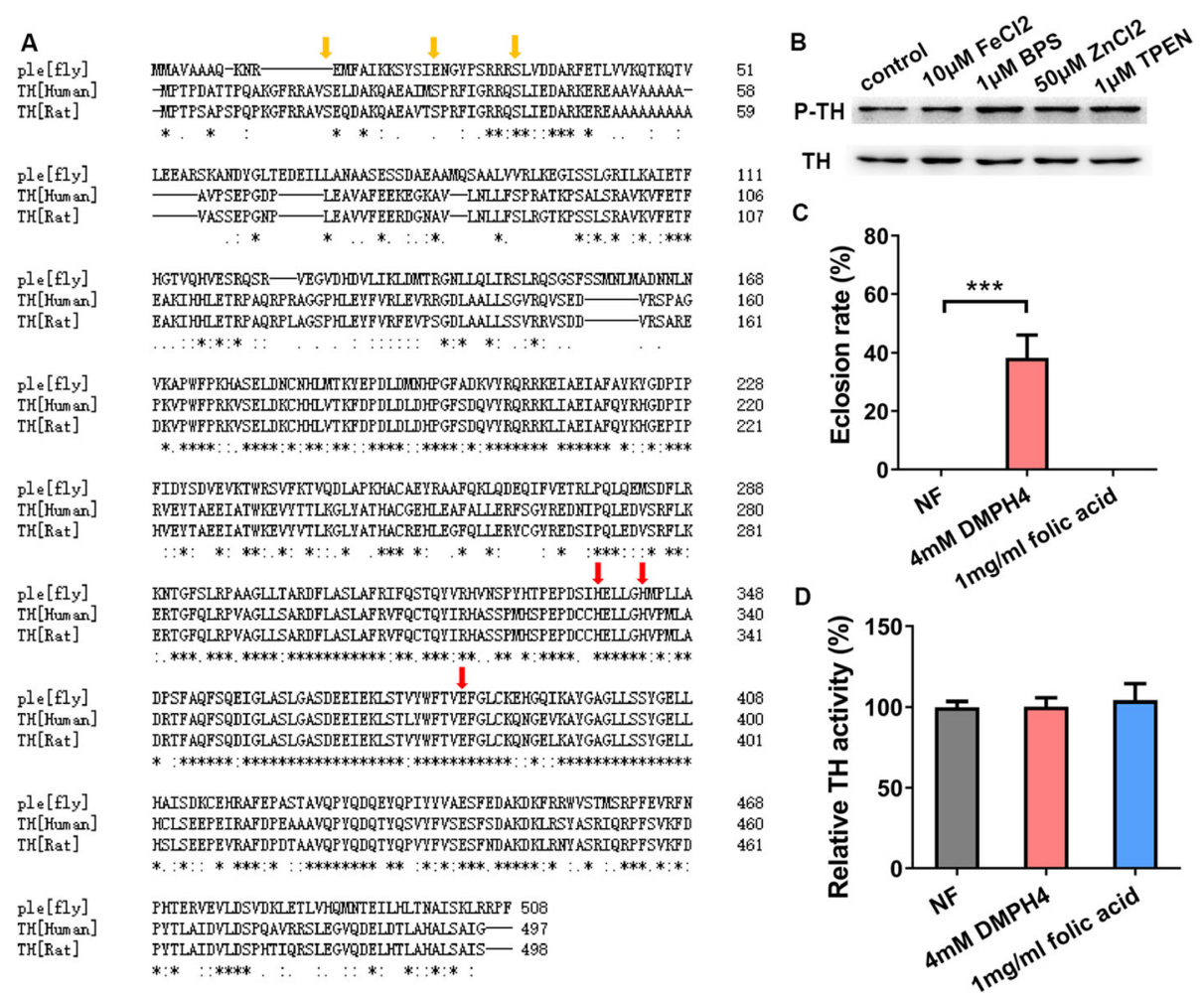

Fig. 4 The in vivo effects of Catsup on $\mathrm{TH}$ appear primarily not due to $\mathrm{TH}$ phosphorylation and $\mathrm{BH}_{4}$ synthesis. A Sequence comparison among the fruit fly, human, and rat THs. Phosphorylation positions in mammalian TH and their corresponding amino acids in Drosophila TH are indicated with yellow arrowheads. Only the critical third phosphorylation site (Ser40) is conserved in the fly. Predicted metal-binding residues are marked with red arrows. B TH phosphorylation in SH-SY5Y cells under different metal or metal chelator treatments. No obvious and consistent changes were observed. An anti-phosphorylated TH antibody was used. Actin was used as a loading control. C The eclosion defect of Da-Gal4>GTPCH RNAi flies was rescued by $\mathrm{DMPH}_{4}$ supplementation in the food. Genotypes of the flies used were Da-Gal4>GTPCH RNAi. All values are presented as mean $\pm \mathrm{SEM} ; n=6$. ${ }^{* * *} p<0.001$. $\mathbf{D} \mathrm{DMPH}_{4}$ supplementation in the food had no effect on the activity of TH. Genotype of the flies used was $W^{1118}$. All values are presented as mean \pm SEM; $n=6$

\section{Zinc and iron antagonize with each other and directly impact TH activity}

Based on our in vivo work as described above, we hypothesized an interaction between zinc and iron in regulating $\mathrm{TH}$ activity. Literature search revealed that it had previously been shown with in vitro biochemistry experiments that iron has a positive effect, while other divalent metal ions such as zinc, cobalt, and nickel ions have negative effects on recombinant human TH activity [52, 53]. However, it is not known whether these metal ions are relevant in vivo in regulating the activity of $\mathrm{TH}$ enzyme, especially when considering that intracellular labile metal ions are normally present at very low concentrations.

We hypothesized that zinc and iron may confer direct and opposite effects on TH within a physiological context. TH is a cytosolic enzyme. From this perspective, the notion that the effect of Catsup mutation on $\mathrm{TH}$ arises from its cytosolic zinc reduction is reasonable. To formally investigate this possibility and exclude other likely interpretations, Catsup was heterologously expressed in yeast cells, a foreign, arguably relatively clean, and physiologically relevant system. Native yeast lacks the TH counterpart as well as $\mathrm{BH}_{4}$ and DA synthesis. We reasoned that if zinc and iron could impact $\mathrm{TH}$ independent of phosphorylation or $\mathrm{BH}_{4}$ variations, we might be able to detect metal-modulated $\mathrm{TH}$ activities in the yeast cells. We first tested whether Catsup indeed functions as a zinc transporter in the yeast. To do that, the ZHY3 strain lacking the Zrt1 and Zrt2 proteins for zinc uptake was used [54]. We considered that by using ZHY3 strain, a zinc-deficient cellular state would be created, and Catsup expression might be able to alleviate this cytosolic zinc deficiency. Interestingly, on the normal media ZHY3 with induced Catsup expression could not grow well (Fig. 5A). This defect could be attributed either to higher zinc in the cytosol or lower zinc in the secretory compartment. However, growth was restored with the cell-permeable zinc chelator TPEN, suggesting the defect arose from zinc accumulation in the cytosol. Consistently, Catsup expression rendered ZHY3 overtly sensitive to zinc stresses (Fig. 5A). These results are consistent with the expected role of Castup in redistributing 

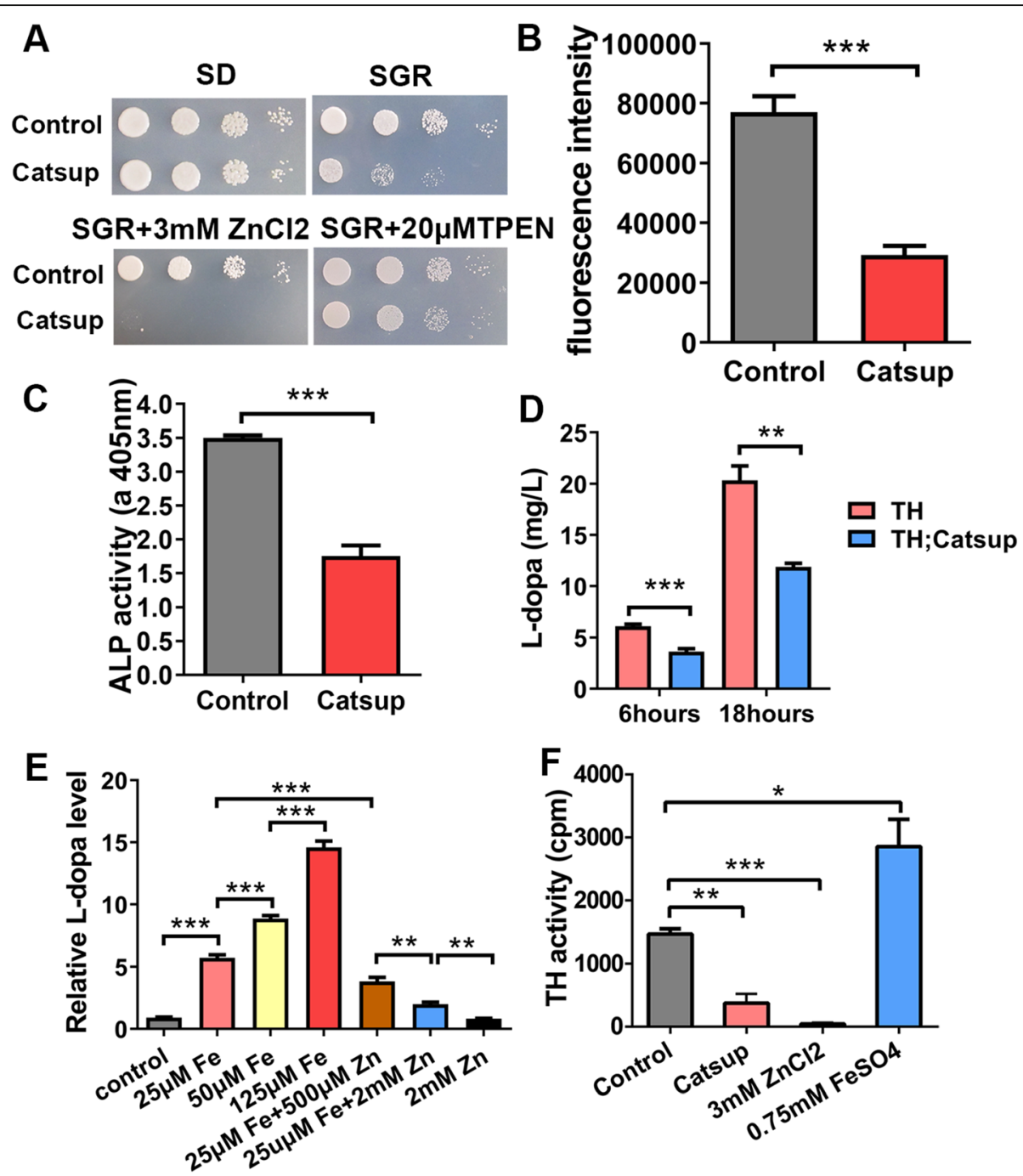

Fig. 5 Zinc and iron potently but oppositely affect TH activity when expressed and assayed in yeast. A The growth defect of ZHY3 yeast expressing Catsup is exacerbated by zinc while rescued by TPEN. Transgenes could be induced on the synthetic galactose and raffinose (SGR) media but not on the synthetic dextrose (SD) media. $n=6$. B The promoter activity of ZRT1 is decreased when Catsup was expressed in ZHY3 yeast. All values are presented as mean \pm SEM and included in Additional file 2: table S3; $n=6 .{ }^{* * *} p<0.001$. C The ALP activity was reduced when Catsup was expressed in $\mathrm{ZHY} 3$ yeast. All values are presented as mean \pm SEM and included in Additional file 2 : table $S 4 ; n=6 .{ }^{* *} p<0.001$. $\mathbf{D}$ L-dopa production in the THexpressing ZHY3 yeast could be inhibited by Catsup expression. Six hours and $18 \mathrm{~h}$ refer to the yeast incubation time for L-dopa production. All values are presented as mean \pm SEM and included in Additional file 2: table $S 5 ; n=4 .{ }^{* *} p<0.01,{ }^{* * *} p<0.001$. E The generation of L-dopa in ZHY3 was elevated by iron and inhibited by zinc added in the media. All values are presented as mean \pm SEM and included in Additional file 2: table S6; $n=3$. ${ }^{* *} p<0.01,{ }^{* * *} p<0.001$. F The activity of TH in ZHY3 was inhibited by Catsup expression or zinc while elevated by iron. All values are presented as mean \pm SEM and included in Additional file 2: table S7; $n=3 .{ }^{*} p<0.05,{ }^{* *} p<0.01,{ }^{* * *} p<0.001$

zinc between the cytosol and the secretory compartments, only the stress in this case primarily arising from zinc accumulation in the cytosol in the presence of toxic amounts of zinc. As an alternative measurement, we used zinc-responsive Zrt1-luciferase reporter to indicate the cytoplasmic zinc pool. Double knockout of Zrt1 and Zrt2 zinc transporter genes results in a low cytoplasmic zinc pool in the yeast cell. When the cytoplasmic zinc pool is low, the transcriptional activity of Zrt1 is upregulated, resulting in a higher luciferase activity. The expression of Catsup in ZHY3 led to a $60 \%$ decrease of luciferase activity compared with that in the control, suggesting an increased zinc level in the cytoplasm (Fig. 5B). The activity of ALP is very sensitive to zinc deficiency [55]. As expected, Catsup expression in ZHY3 significantly reduced the activity of ALP, indicating a decreased zinc level in the secretory pathway (Fig. 5C).

To analyze zinc's effect on the activity of TH in yeast, Drosophila TH was then introduced into the ZHY3 strain. The yeast was incubated in the presence of $1 \mathrm{mM}$ $\mathrm{DMPH}_{4}$ as yeast lacks endogenous $\mathrm{BH}_{4}$ synthesis. As 
shown in Fig. 5D, ZHY3 expressing TH synthesized Ldopa, and the expression of Catsup in ZHY3 resulted in a $40 \%$ decrease of the L-dopa level. We then tested the influence of exogeneous zinc and iron on the $\mathrm{TH}$ activity by measuring L-dopa production (Fig. 5E). The amount of L-dopa synthesized in ZHY3 expressing TH increased with iron and decreased with zinc supplementation (Fig. $5 \mathrm{E})$. We also directly measured the $\mathrm{TH}$ activity with the radioisotope (Fig. 5F). The results indicated that the $\mathrm{TH}$ activity was inhibited by Catsup and zinc, whereas induced by iron (Fig. 5F). Given the unexpected growth inhibitory effect of Catsup on ZHY3, the effect of zinc and iron on Ldopa synthesis was further examined with wild-type INVSc1 strain expressing $\mathrm{TH}$, and similar results were obtained (Additional file 1: Figure S2). These results indicated that the activity of $\mathrm{TH}$ heterologously expressed in the yeast can be influenced by the intracellular iron or zinc available to cells. Noteworthy is that since the yeast is unable to synthesize $\mathrm{BH}_{4}$, the fact that under a fixed $\mathrm{BH}_{4}$ level zinc and iron could still markedly affect $\mathrm{TH}$ activity indicates that the zinc and iron effect on $\mathrm{TH}$ could be direct.

We also tested $\mathrm{TH}$ responsiveness to iron and zinc in E. coli. Native E. coli also lacks TH and DA synthesis, as well as the essential cofactor $\mathrm{BH}_{4}$. Drosophila $\mathrm{TH}$ was expressed in $E$. coli and provided with exogenous $\mathrm{DMPH}_{4}$. As shown in Fig. 6A, E. coli-expressing Drosophila TH synthesized L-dopa, and the amount of Ldopa synthesized increased with incremental elevations of iron and decreased with zinc. The expression of $\mathrm{TH}$, on the other hand, appeared not much affected by the addition of zinc and iron (Additional file 1: Figure S3). Therefore, both the experiments in the yeast and E. coli indicate that TH activities within a cell could be modulated robustly and possibly directly by iron and zinc homeostasis, and that iron and zinc antagonize each other in regulating $\mathrm{TH}$ activity.

It is known that iron is a cofactor of $\mathrm{TH}$ and the redox state of the iron is essential for its function [52]. Sitedirected mutagenesis studies and crystal structure analysis revealed that the side chains of two histidine residues and one glutamate residue in the active site of mammalian $\mathrm{TH}$ are directly involved in iron coordination [56]. Some other divalent metal ions also could bind with $\mathrm{TH}$ and inhibit its activity in vitro $[52,53]$. Not surprisingly, Drosophila $\mathrm{TH}$ and human $\mathrm{TH}$ are well conserved in these ironbinding sites (Fig. 4A). We hypothesized zinc could compete with iron binding with Drosophila TH and subsequently inhibit its activity. To this end, we performed a metal-binding assay. Drosophila TH was expressed in $E$. coli and purified. The purified recombinant Drosophila $\mathrm{TH}$ was incubated with $\mathrm{Fe}^{2+}$ and $\mathrm{Zn}^{2+}$. The bound and free iron fractions were then separated and measured by BPS-based colorimetric assays (Fig. 6B). We observed that Drosophila TH contained about 0.8 iron atom/subunit when incubated with ferrous iron, and the amount of bound iron decreased with increasing concentrations of zinc in the incubation buffer (Fig. 6B). The competing binding of iron and zinc on $\mathrm{TH}$ was further confirmed by ICP-MS (Additional file 1: Figure S4). Notably, zinc appears to be able to bind $\mathrm{TH}$ at multiple sites, likely as a result of non-specific binding in particular at high concentrations. These results indicate that zinc regulates Drosophila $\mathrm{TH}$ activity by affecting $\mathrm{TH}$ binding with iron.

\section{Modulation of zinc and iron homeostasis alters phenotypes associated with PD}

$\mathrm{PD}$ is a neurodegenerative disorder characterized in its late phase by sustained loss of dopaminergic neurons

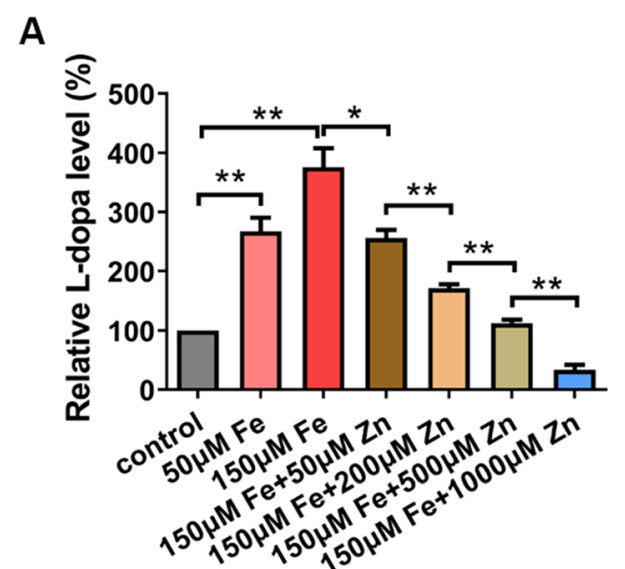

B

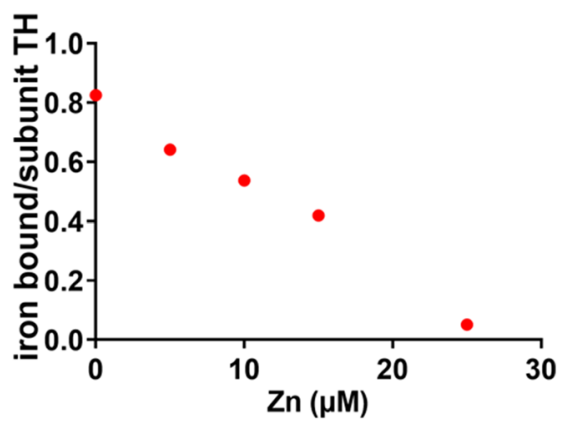

Fig. 6 Zinc ion interferes with ferrous iron binding to TH. A The production of L-dopa in E. coli-expressing TH was elevated by iron and inhibited by zinc added in the media. All values are presented as mean \pm SEM and included in Additional file 2 : table S8; $n=3$. ${ }^{*} p<0.05,{ }^{* *} p<0.01$. $\mathbf{B}$ Binding of iron to Drosophila TH. Apo-TH was incubated with $15 \mu \mathrm{M}$ ferrous iron and different concentrations of zinc. Free and bound metal ions were separated by rapid ultrafiltration, and the iron level was determined by BPS-based colorimetric assays. The amount of bound iron decreased with increased zinc concentration in the buffer. All values presented are included in Additional file 2: table S12 
from the substantia nigra pars compacta $(\mathrm{SNpc})$ and other brainstem regions [57]. Since DA loss is a cardinal feature of PD and L-dopa is utilized as an initial therapy to treat PD, we wondered whether the effect of zinc and iron on TH could influence PD phenotypes. Rotenone is a specific high-affinity inhibitor of mitochondrial complex I [58] and has been shown to be an environmental toxin (along with other chemicals such as MPTP and paraquat) contributing significantly towards the occurrence of sporadic PD in humans and animal models [59]. Rotenone exposure caused serious mobility impairment and reduced lifespan for Drosophila at $25^{\circ} \mathrm{C}$ (Fig. $7 \mathrm{~A}, \mathrm{~B})$. When raised at $25^{\circ} \mathrm{C}$ for 7 days in the presence of rotenone, about $30 \%$ of flies reached the subjectively designated height $(8 \mathrm{~cm})$ within a set time $(7 \mathrm{~s})$ (Fig. 7A). Catsup RNAi and $d Z n T 7$ OE were significantly rescued, whereas Catsup OE exacerbated, the mobility impairment (Fig. 7A). Consistent with the results described above, Catsup RNAi and $d Z n T 7$ OE extended the reduced longevity of rotenone-treated flies, whereas Catsup OE shortened it (Fig. 7 B). The overexpression of Malvolio $(\mathrm{Mvl})$, an iron importer which presumably increases the iron level in the cytoplasm, also exhibited dramatic rescuing effects on the mobility impairment and the reduced lifespan (Fig. 7A, B). Catsup RNAi, $d Z n T 7$ OE, Catsup OE, and $M v l$ OE all exhibited mobility and survival defects when raised on normal food (Additional file 1: Figure S5). However, their effects on roteone-induced PD are diverse. Catsup OE, which on normal food survived better than Catsup RNAi and $d Z n T 7$ OE (Additional file 1: Figure S5), faired much worse on rotenone (Fig. 7A, B). In fact, although Catsup RNAi and $d Z n T 7$ OE were harmful on their own in the normal context (Additional file 1: Figure S5), they
A
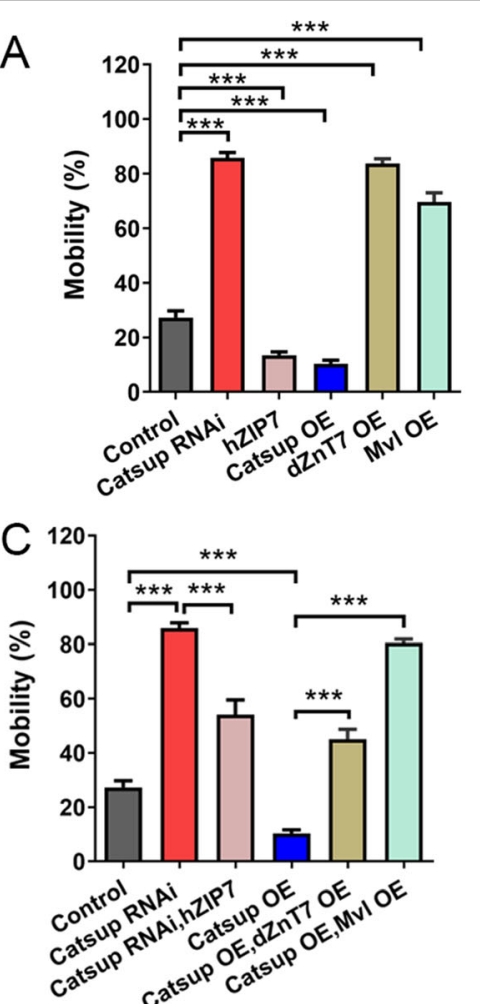

B

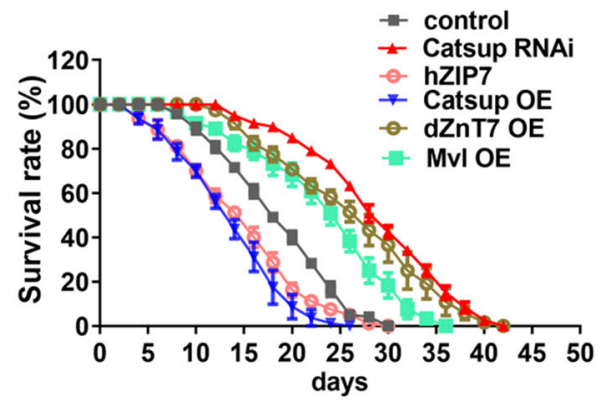

D

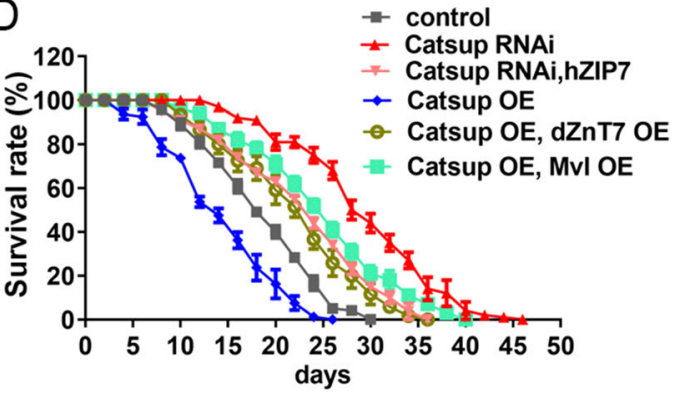

Fig. 7 Modulation of zinc and iron homeostasis is an effective means to alter a spectrum of phenotypes associated with PD. A The mobility defect of PD flies (exposing to rotenone) could be rescued by Catsup RNAi, dZnT7 OE, or Mvl OE. Genotypes of the flies used were TH-Gal4>W ${ }^{1718}$ (control), TH-Gal4>Catsup RNAi, TH-Gal4>Catsup OE, TH-Gal4>dZnT7 OE, and TH-Gal4>Mvl OE. All values are presented as mean \pm SEM and included in Additional file 2: table S9; $n \geq 6$. ${ }^{* *} p<0.001$. B The shortened lifespan of PD flies (exposing to rotenone) could be modified by adjusting zinc transporter expressions. Genotypes of the flies used were TH-Gal4>W ${ }^{1178}$ (control), TH-Gal4>Catsup RNAi, TH-Gal4>Catsup OE, THGal4>dZnT7 OE, and TH-Gal4>MVI OE. C The mobility rescue effect of Catsup RNAi on PD flies (exposing to rotenone) could be neutralized by hZIP7 expression. The exacerbated defect of Catsup OE on PD flies could be rescued by dZnT7 OE and Mvl OE. Genotypes of the flies used were TH-Gal4>w W $^{1718}$ (control), TH-Gal4>Catsup RNAi, TH-Gal4>Catsup RNAi, hZIP7 OE, TH-Gal4>Catsup OE, TH-Gal4> Catsup OE; dZnT7 OE, TH-Gal4> Catsup $O E$, and MVI OE. All values are presented as mean \pm SEM and included in Additional file 2 : table S10; $n \geq 6 .{ }^{* * *} p<0.001$. One-way analysis of variance (ANOVA) was used for multiple groups. D The lifespan rescue of Catsup RNAi on PD flies (exposing to rotenone) could be neutralized by hZIP7 expression. The exacerbated defect of Catsup OE on PD flies could be rescued by dZnT7 OE and Mvl OE. Genotypes of the flies used were TH-Gal4>w ${ }^{1118}$ (control), TH-Gal4>Catsup RNAi, TH-Gal4>Catsup RNAi, hZIP7 OE, TH-Gal4>Catsup OE, TH-Gal4> Catsup OE, dZnT7 OE, TH-Gal4> Catsup OE, and MVI OE. All values are presented as mean \pm SEM and included in Additional file 2 : table $S 11 ; n \geq 4 .{ }^{* *} p<0.001$ 
displayed a strong rescuing effect on rotenone-treated flies (Fig. 7A, B).

Further study indicated that the rescue effect of Catsup RNAi on flies with rotenone exposure could be inhibited by hZIP7 OE, and the exacerbation effect of Catsup OE on these flies could be rescued by dZnT7 OE and $M v l$ OE (Fig. 7C, D). Taken together, these results indicated that modulating the zinc and iron levels in the Drosophila brain could modify a whole range of symptoms of rotenone-treated flies.

\section{Discussion}

Zinc and iron are critical to a variety of proteins and are essential for normal CNS development and function [60]. Previous studies showed that Catsup mutants have significantly elevated $\mathrm{TH}$ activity, resulting in abnormally high levels of catecholamines [19]. In this study, we confirmed that Catsup is indeed functionally analogous to hZIP7, transporting zinc from the secretory compartments into the cytoplasm. We found that manipulating Catsup in the brains can greatly modulate DA synthesis and PD progress by modulating $\mathrm{TH}$ activity through the interference of iron. We provided in vivo evidence that $\mathrm{TH}$ activity is affected by a balance of zinc and iron levels. Further study indicated that manipulation of zinc levels and iron levels in PD brains may be a novel therapeutic strategy. This may hold great significance for the prevention and treatment of PD and even other diseases caused by catecholamine dysfunction.

Ubiquitous manipulation of zinc and iron homeostasis often resulted in reduced viability. For this reason, dramatic modulation of metal homeostasis may not be feasible in PD rescue. We have primarily relied on genetic measures to modulate regional metal homeostasis such as in the brain or TH-positive cells to achieve a significant effect. It is envisioned that in clinical cases, targeted modification of metal homeostasis may be necessary to obtain satisfactory results. This is particularly so when the blood-brain barrier is taken into account, which may partially insulate the brain from the circulation system. Indeed, in our case, the nutritional supplement of metals in the food was much less efficient to reach the kind of rescue we saw with tissue-specific genetic interventions.

Catsup is a member of the ZIP (Slc39A) transporter family [30]. Most ZIP family members transport zinc, but a few have been found to transport other metals, including ZIP8, ZIP13, and ZIP14 [61]. Catsup shares the highest overall homology with human ZIP7 $[25,30]$, which is an identified zinc transporter [26]. Simultaneously, Catsup also shares high homology with human dZIP13, which fulfills the iron effluxing role in Drosophila [30]. In this study, we studied the function of Catsup and verified that it is a zinc transporter located in the secretory compartments. In a previous study, we provided in vivo functional analysis to differentiate different transporting functions of these two ZIPs; the HNXXD motif is required for zinc transport activities for Catsup [37].

The activity of many enzymes is regulated by metal ions. For example, the activity of superoxide dismutase (SOD) can be induced by copper but inhibited by iron or cadmium [62]; cytochrome c oxidase needs iron and copper to execute its activity, but the presence of zinc inhibits its activity [63]. So, it seems important to balance these metal ions in vivo to regulate the activity of these enzymes. In this study, we showed that in vivo iron stimulates while zinc inhibits $\mathrm{TH}$ activity, consistent with the previous report that the activity of recombinant human $\mathrm{TH}$ was rapidly activated by incubation with iron and inhibited by some other metal ions in vitro [52].

Protein phosphorylation is reported as one major mechanism for controlling the activity of $\mathrm{TH}$ [43]. TH phosphorylation is regulated by several protein kinases and protein phosphatases [43]. It is well known that zinc plays an important role in affecting phosphorylation signaling $[64,65]$. Here, we found that the TH phosphorylation is not appreciably affected by zinc or iron modulation, so the effect of Catsup on $\mathrm{TH}$ activity seems through a route other than phosphorylation.

$\mathrm{TH}$ requires $\mathrm{BH}_{4}$ as a cofactor [48], and the enzyme activity is catalyzed by ferrous iron [66]. As a result, the synthesis of catecholamines is not only dependent on $\mathrm{TH}$ activity but also on the level of $\mathrm{BH}_{4}$ and iron. Because the $\mathrm{TH}$ activity is not increased in flies feeding with $\mathrm{DMPH}_{4}$, we proposed that under normal conditions, the $\mathrm{BH}_{4}$ level in the body may be sufficient or saturated, so that excess $\mathrm{BH}_{4}$ in vivo has no further contribution to the $\mathrm{TH}$ activity. Expression of $\mathrm{TH}$ within the yeast and $E$. coli, both of which lack $\mathrm{BH}_{4}$ synthesis and likely some other regulatory pathways tuning $\mathrm{TH}$ activity, indicated that $\mathrm{TH}$ activity is robustly responsive to iron and zinc modulation. Altogether, we propose that iron in the cytoplasm binds $\mathrm{TH}$ to catalyze its activity, and zinc can compete with iron binding in TH. Since Catsup is responsible for zinc release from the secretary pathway to cytoplasm, Catsup mutations lead to zinc deficiency in the cytoplasm, and in consequence, more iron could bind to $\mathrm{TH}$, resulting in the increased $\mathrm{TH}$ activity (Fig. 8).

Increasing amounts of evidence show that imbalance of trace metal homeostasis is strongly related to the pathological process of PD $[67,68]$. Here, we revealed that there exists a new way to regulate DA synthesis in vivo through modulating metal homeostasis. The knowledge gained in this study appeared to be adaptable in ameliorating PD features in a fly model. It is reasonable to speculate that cells could physiologically modulate dopamine synthesis through zinc control, by 


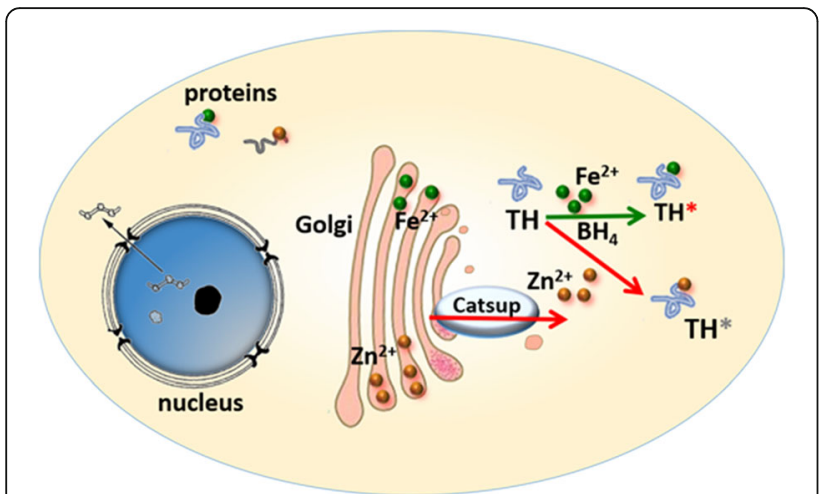

Fig. 8 A model to explain Catsup's effect on TH activity. Zinc appears to be an important regulator of $\mathrm{TH}$ activity or dopamine production in vivo. When Catsup is mutated, zinc fails to be effectively redistributed between ER/Golgi and cytosol, leading to zinc elevation in the secretory pathway but reduction in the cytosol. Zinc normally antagonizes iron in regulating TH activity. When zinc release is blocked, TH has better access to iron and its activity is elevated

maintaining a balance between zinc storage and release to adjust the cytosolic zinc levels. Indeed, mammalian ZIP7 was reported to be regulated by phosphorylation [69]. Therefore, it is expected that cells could adjust DA levels through modifying expression or posttranslational modifications of ZIP7 in response to physiological changes.

These results may suggest new strategies for the prevention and treatment of PD and some other diseases closely related to DA. Considering zinc depletion and iron increase may achieve similar end results, in our opinion, it may be preferable to use iron limitation strategy when depressing dopamine levels is intended or zinc limitation in the case of boosting dopamine production. This is because iron may in short term raise dopamine levels; it may also simultaneously stimulate elevated production of reactive oxygen species, heightening dopaminergic neuron damages in the long run. Besides PD, DA is closely related to pain, drug addiction, attention deficit hyperactivity disorder, and many kinds of mental diseases such as psychosis, schizophrenia, and depression $[2,5]$. Unfortunately, the etiology of many of these diseases remains less well clarified. It would be interesting to see how the balance of zinc and iron could modulate the disease progression for some of these physiological disorders that might be DA-related.

\section{Conclusions}

Analyses of dopamine elevation in Catsup mutation/ knock-down revealed an important role of zinc homeostasis in regulating $\mathrm{TH}$ activity. Zinc antagonizes ferrous iron, a cofactor of the TH enzyme, to exert a negative effect on TH. This work enriches our understanding of dopamine regulation in vivo and may help our fight against PD and other forms of dopamine-related diseases.

\section{Methods \\ Drosophila strains, yeast strains, and maintenance}

Drosophila strains used in this study are listed as follows: Da-Gal4 (Bloomington \#8641), Elav-Gal4 (Bloomington \#458), and TH-Gal4 (Bloomington \#8848), all from the Bloomington Drosophila Stock Center. $p$ Catsup RNAi (VDRC \#100095) and dZnT7 RNAi (VDRC \#107388) were obtained from the Vienna Drosophila RNAi Center. Transgenic fly strains used in this report have been previously described, including $d Z I P 13 \mathrm{OE}$ [37], dZIP1 OE [42], dZnT1 OE [39]. pUAST-Malvolio construct $(M v l \mathrm{OE})$ was injected into $w^{1118}$ background flies following standard protocols. One line with relatively more severe neuronal phenotype when directed by ELav-Gal4 was chosen for the studies reported here.

All flies were maintained on standard cornmeal media at $25{ }^{\circ} \mathrm{C}$ with $60 \%$ humidity under a 12-h light-dark cycle unless otherwise stated. Concentrations of supplemented metals or metal chelators used were as follows: $5 \mathrm{mM}$ ferric ammonium citrate (FAC; Sigma-Aldrich), $150 \mu \mathrm{M}$ bathophenanthroline disulfonate (BPS; SigmaAldrich), $50 \mu \mathrm{M} \quad N, N, N^{\prime}, N^{\prime}$-tetrakis (2-pyridylmethyl) ethylenediamine (TPEN; Sigma-Aldrich), and $2 \mathrm{mM}$ $\mathrm{ZnCl}_{2}$ (Beijing Yili Fine Chemicals Ltd. Co., Beijing, China). For inhibitor studies, concentration of 3-Iodo- $L$ tyrosine (3-IT; Sigma-Aldrich) used was $75 \mu \mathrm{M}$. For the rotenone feeding experiment, 2-day-old adult flies were transferred to vials with instant Drosophila medium and exposed to $150 \mu \mathrm{M}$ freshly prepared rotenone (SigmaAldrich) for 7 days. The medium was replaced every day to ensure that the rotenone was effective.

The ZHY3 strain lacking the Zrt1 and Zrt2 proteins for zinc uptake was used in this study [54].

\section{Cell culture and plasmid transfection}

For subcellular localization experiments, CHO-K1 cells were maintained in DMEM (Invitrogen) containing 10\% fetal bovine serum (FBS, Gibco BRL, Gaithersburg, MD, USA) at $37^{\circ} \mathrm{C}$ under $5 \% \mathrm{CO}_{2}$ atmosphere. When the cells reached $80 \%$ confluence, they were transfected with pIRESneo-Catsup-FLAG using Lipofectamine ${ }^{\mathrm{Tm}} 3000$ (Invitrogen) in accordance with the manufacturer's instructions. After $24 \mathrm{~h}$, cells were fixed, stained with the FLAG antibody (1:500) and the Golgi marker (antiGM130, 1:500) or ER marker (anti-PDI, 1:500), and imaged with the ZEISS LSM780 microscope. Anti-FLAG mouse monoclonal antibody (ab18230), anti-FLAG rabbit polyclonal antibody (ab1162), anti-GM130 rabbit polyclonal antibody (ab30637), and anti-PDI mouse monoclonal antibody (ab2792) were obtained from 
Abcam (Cambridge, MA, USA). Secondary antibodies include cy3-conjugated goat anti-mouse and cy3conjugated goat anti-rabbit IgG (Zhongshan Goldenbridge Biotechnology, Beijing, China). The experiments were repeated three times.

SH-SY5Y cells were used to test the effect of metals on mammalian TH phosphorylation at Ser40. SH-SY5Y cells were maintained in DMEM (Invitrogen) with 10\% FBS and 1\% PS under an atmosphere of 95\% air and 5\% $\mathrm{CO}_{2}$ at $37^{\circ} \mathrm{C}$. To test metals' effects, $1 \mu \mathrm{M}$ BPS, $50 \mu \mathrm{M}$ $\mathrm{ZnCl}_{2}, 1 \mu \mathrm{M}$ TPEN, and $10 \mu \mathrm{M} \mathrm{FeCl}{ }_{2}(2 \mathrm{mg} / \mathrm{mL}$ vitamin $\mathrm{C}$ were added in the medium to inhibit iron oxidation) were respectively added in the medium. The experiments were repeated three times.

\section{Eclosion, mobility, and longevity assays}

For eclosion assays, Gal4 lines were crossed with various transgenic lines for $\sim 3$ days, as indicated in each experiment, and allowed to lay eggs on juice-agar plates for $\sim 24$ h. Newly hatched first-instar larvae were transferred to normal food or food supplemented with metals or metal chelators, as indicated. The density of each vial was controlled to 60-100 larvae, and the total number of emerging adults of each genotype was counted [30]. Assays were done with six replicates for each group, and the experiments were repeated at least three times. The results from all experiment runs were pooled together for analyses.

To assay the mobility of PD flies, 20 females were placed in a vertical glass vial. After a 1-h recovery from $\mathrm{CO}_{2}$ exposure, flies were gently tapped to the bottom of the column. The number of flies reaching the indicated height $(\sim 8 \mathrm{~cm})$ was counted after $7 \mathrm{~s}$ of climbing under red light. Six parallel group tests were conducted for each genotype, and the experiments were repeated at least three times [34]. The presented data are from the results of all experiments.

For longevity assays, 3-day-old adult males or females were kept in vials (20 individuals of each vial) containing cornmeal medium and maintained at $25^{\circ} \mathrm{C}$. Flies were transferred to fresh media every $2-3$ days, and the number of live flies was recorded every alternate day. Six parallel group tests were conducted for each genotype, and the experiments were repeated at least three times [34]. The data were presented as Kaplan-Meier survival distributions, and significance was determined by log-rank tests.

\section{Eye morphology analysis}

To phenotype eye morphology, flies were collected after eclosion at $18{ }^{\circ} \mathrm{C}$ and frozen to death at $-80^{\circ} \mathrm{C}$ for one night. The eyes were photographed using a Zeiss imager A1 stereomicroscope. More than six flies were scored per genotype, and each experiment was repeated three times. Scanning electron microscopy analysis of fly eyes was performed as described previously with FEI Quanta 200 [70].

\section{Alkaline phosphatase(ALP) activity assay}

ALP activity assays were performed as described previously with some modifications [71]. For yeast samples, yeast was cultured in synthetic dextrose media (SD) at $30{ }^{\circ} \mathrm{C}$ to $\mathrm{OD}=0.4$, collected by centrifugation at $3000 \mathrm{~g}$ for $5 \mathrm{~min}$, and then transferred to the induction medium (synthetic glucose and raffinose (SGR) media). The yeast was cultured in SGR media at $30{ }^{\circ} \mathrm{C}$ to $\mathrm{OD}=1$, collected by centrifugation, and washed three times with ice-cold PBS. The collected yeast was homogenized in ALP lysis buffer $\left(1.0 \mathrm{mM}\right.$ Tris- $\mathrm{HCl} \mathrm{pH} \mathrm{7.4,} 0.5 \mathrm{mM} \mathrm{MgCl}_{2}$, and $0.1 \%$ Triton $\mathrm{X}-100$ ), and the protein concentration was measured by the BCA kit (Thermal). Approximately $2 \mu \mathrm{g}$ protein was added to $90 \mu \mathrm{L}$ solution A (1.0 M diethanolamine, $0.5 \mathrm{mM} \mathrm{MgCl}_{2} \mathrm{pH} 9.8$ ) and $10 \mu \mathrm{L}$ solution B (150 mM p-nitrophenyl phosphate). After incubation for $30 \mathrm{~min}$ at $30^{\circ} \mathrm{C}$, ALP activity was measured based on the p-nitrophenol release by its absorbance at $405 \mathrm{~nm}$.

For fly samples, $\sim 80$ adult heads were lysed in the ALP lysis buffer, then $\sim 2 \mu \mathrm{g}$ protein was added to $90 \mu \mathrm{L}$ solution A and $10 \mu \mathrm{L}$ solution B. The absorbance at 405 $\mathrm{nm}$ was measured after incubation for $30 \mathrm{~min}$ at $25^{\circ} \mathrm{C}$. The experiments were repeated three times.

\section{Luciferase activity assay}

Yeast was cultured in SD media at $30{ }^{\circ} \mathrm{C}$ to $\mathrm{OD}=0.4$, collected by centrifugation at $3000 \mathrm{~g}$ for $5 \mathrm{~min}$, and then transferred to SGR media. The yeast was cultured in SGR media at $30{ }^{\circ} \mathrm{C}$ to $\mathrm{OD}=1$, collected by centrifugation, and washed three times with ice-cold PBS. The collected yeast was homogenized in cold PBS, and the protein concentration was measured by the BCA kit (Thermal). Approximately $10 \mu \mathrm{g}$ protein was used to test the luciferase activity by Firefly Luciferase Reporter Gene Assay Kit (Beyotime, \#RG027); fluorescence intensity was measured for $10 \mathrm{~s}$ by the fluorescence microplate reader. In order to avoid the error caused by the difference in the amount of samples, the reporter gene of Renilla luciferase was used as an internal reference.

\section{Western blot analysis}

SH-SY5Y cells were used to test the effect of metals on mammalian TH phosphorylation at Ser40. Antibodies of anti-tyrosine hydroxylase (AB152) and anti-tyrosine hydroxylase, phosphoSer 40 (AB5935) were purchased from Sigma-Aldrich (Shanghai, China). The secondary antibody HRP-conjugated goat anti-rabbit IgG was purchased from Zhongshan Goldenbridge Biotechnology (Beijing, China). For Western blot analysis, cells were homogenized in the buffer containing 1\% Triton X-100 plus $10 \%$ proteinase inhibitor cocktail (Sigma), centrifuged, separated on $10 \%$ SDS-PAGE, and transferred to nitrocellulose membranes (Millipore, Watford, UK). Signals were developed with the ECL detection kit 
(Vigorous Biotechnology, Beijing, China). The experiments were repeated three times.

\section{Metal sensitivity/resistance assay in yeast}

Catsup was cloned into pYES2 (Invitrogen) and transformed into ZHY3. The vector pYES2 was also transformed into yeast and used as the control. For growth testing on agar plates (spotting assay), yeast grown in the culture medium (synthetic dextrose media, SD) was 10-fold serially diluted with sterile $\mathrm{ddH}_{2} \mathrm{O}$ and then spotted on SD (the transgene could not be induced on this medium) or synthetic galactose and raffinose media (SGR, the transgene could be induced on this medium) plates with or without metal or chelators. Incubation was all at $30^{\circ} \mathrm{C} ; 3 \mathrm{mM} \mathrm{ZnCl}{ }_{2}$ or $20 \mu \mathrm{M}$ TPEN was added in the medium, $n=6$.

\section{Metal ions binding assays of $\mathrm{TH}$}

A double-tagged Drosophila $\mathrm{TH}$ protein (dTH) expression plasmid containing N-terminal His6 and SUMO tag (pET28a-His6-SUMO-dTH) was constructed. The ensuing plasmid was transformed into E. coli BL21(DE3). Protein expression was induced by $250 \mu \mathrm{M} \beta$-D-1-thiogalactopyranoside (IPTG) with $100 \mu \mathrm{M} \mathrm{FeSO} 4$ supplied in LB medium for enzyme stability. Induction proceeded at $16^{\circ} \mathrm{C}$ for $14 \mathrm{~h}$. The His6-SUMO-dTH protein was purified with Ni-NTA resin, and the tags were removed by ULP1 digestion. Protein was then loaded onto a Superdex 200 gel filtration column for size separation. The protein composition of each fraction was determined by SDS-PAGE analysis. Fractions containing dTH were collected, and EDTA was added to a final concentration of $1 \mathrm{mM}$ to remove divalent metal ions. After incubation at $4{ }^{\circ} \mathrm{C}$ for $30 \mathrm{~min}$, the purified protein was concentrated by Amicon ${ }^{\circ}$ Ultra Centrifugal Filters (type 30,000 NMWL). Then, the protein was diluted 10-fold with EDTA-free $50 \mathrm{mM}$ MES buffer ( $\mathrm{pH}$ 6.5) and applied to Ultra filters again to be concentrated to its original volume. The procedure was repeated for five times to remove EDTA in the protein buffer.

Competitive binding assays were performed as described previously with some modification [52]. Briefly, purified dTH $(2.5 \mu \mathrm{M}$ subunit) was incubated with $15 \mu \mathrm{M} \mathrm{FeCl} 2,0-25 \mu \mathrm{M} \mathrm{ZnCl}_{2}$, and $0.5 \mathrm{mg} / \mathrm{mL}$ catalase in $50 \mathrm{mM}$ MES Potassium (pH 6.5) for $20 \mathrm{~min}$ at $4{ }^{\circ} \mathrm{C}$. After incubation, the mixture was applied to Amicon Ultra filter devices (Merck Millipore). Free and bound metal ions were separated by centrifugation ( $5 \mathrm{~min}$ at 5000 $\mathrm{rpm})$ at $4{ }^{\circ} \mathrm{C}$. The filtrate was collected and contents of iron ion or zinc ion were determined by BPS-based colorimetric assays or ICP-MS. All the experiments were repeated at least three times.

\section{Determination of L-dopa}

For L-dopa assay in S. cerevisiae, Catsup and dTH were cloned into pYES2 (Invitrogen) and pYES3 (Invitrogen), respectively. The plasmids were transformed into $\mathrm{ZHY} 3$ or wild-type yeast INVSc1. The vectors pYES3 and pYES2 were also transformed into yeast and used as the controls. For L-dopa produced in yeast, a single colony was picked and grown in $\mathrm{SD}$ medium at $30{ }^{\circ} \mathrm{C}$ to $\mathrm{OD}_{600}=1$. Then, the yeast cells were collected by centrifugation at $3000 \mathrm{~g}$ for $5 \mathrm{~min}$, washed with sterile water, and transferred to SGR medium supplemented with $0.4 \mathrm{mg} / \mathrm{mL}$ tyrosine, 2.0 $\mathrm{mg} / \mathrm{mL}$ ascorbic acid, and $1 \mathrm{mM} \mathrm{DMPH}_{4}$. In the experiments for testing metal effects, $400 \mathrm{mM}$ BPS along with $\mathrm{FeSO}_{4}$ or $\mathrm{ZnCl}_{2}$ was added in the medium. The yeast was shaken at $30{ }^{\circ} \mathrm{C}$ for $18 \mathrm{~h}$, and the supernatant was collected for L-dopa level determination.

For L-dopa assays in E.coli, pET28a-His6-SUMO-dTH mentioned above was transformed into BL21 (DE3). The vector pET28a was transformed into BL21(DE3) as control. Protein expression was induced by $250 \mu \mathrm{M}$ IPTG at $37^{\circ} \mathrm{C}$. The LB medium was supplied with $400 \mu \mathrm{g} / \mathrm{mL}$ tyrosine, $2.0 \mathrm{mg} / \mathrm{mL}$ ascorbic acid, and $1 \mathrm{mM} \mathrm{DMPH}_{4}$ for L-dopa production. $\mathrm{FeSO}_{4}$ or $\mathrm{ZnCl}_{2}$ was added as indicated in each experiment. L-dopa level was determined after $8 \mathrm{~h}$ growth at $37^{\circ} \mathrm{C}$. L-dopa level was determined as previously described [72] with some modifications. Briefly, the colorimetric method for estimation of LDOPA was performed in 96-well microtiter plates. Fifty microliters each of $0.5 \mathrm{M} \mathrm{HCl}$, nitrite-molybdate reagent (composed of $10 \% \mathrm{w} / \mathrm{v}$ sodium nitrite and $10 \% \mathrm{w} / \mathrm{v}$ sodium molybdate), and $1.0 \mathrm{M} \mathrm{NaOH}$ was sequentially added to $50 \mu \mathrm{L}$ of the sample. The absorbance was measured at $530 \mathrm{~nm}$ using a Thermo Multiskan GO reader. A calibration curve with different amounts of L-dopa was constructed. L-dopa level in each sample was calculated based on the standard curve or normalized to the control. All experiments were repeated at least three times.

\section{Dopamine level determination}

For sample preparation, 3rd larvae were collected and sonicated in $0.6 \mathrm{~mL}$ of ice-cold $0.4 \mathrm{M}$ perchloric acid. After centrifugation at $13000 \mathrm{~g}$ at $4{ }^{\circ} \mathrm{C}$ for $10 \mathrm{~min}$, the supernatant was transferred into a new tube and mixed with a half supernatant volume of $20 \mathrm{mM}$ potassium citrate, $300 \mathrm{mM} \mathrm{K} \mathrm{HPO}_{4}$, and $2 \mathrm{mM}$ EDTA. The mixture was kept on ice in the dark for $20 \mathrm{~min}$. Then, the chilled mixture was filtered through a low-binding Durapore $(0.22 \mu \mathrm{m})$ PVDF membrane. The amounts of dopamine were measured by an HPLC-ECD system. Chromatographic separation was performed on a Diamonsil C18 column $(5 \mu \mathrm{m}, 250 \times 4.6 \mathrm{~mm})$. The mobile phase contained $\quad 60 \mathrm{mM} \quad \mathrm{KH}_{2} \mathrm{PO}_{4}, \quad 350 \mu \mathrm{M} \quad \mathrm{C}_{8} \mathrm{H}_{19} \mathrm{NaO}_{4} \mathrm{~S}$, $100 \mu \mathrm{M}$ EDTA, and $8 \%$ formaldehyde. Dopamine levels 
were monitored using a HPLC system (Water e2695) equipped with an electrochemical detector (Water 2645). The experiments were repeated three times.

\section{TH activity assay}

$\mathrm{TH}$ activity was determined as previously described with some modifications [73, 74]. For the TH activity assay in Drosophila heads, samples were collected and homogenized in $20 \mathrm{mM}$ Tris- $\mathrm{HCl}$ buffer, $\mathrm{pH} 7.5$ by ultrasonic disintegration over ice. Protein concentrations were determined with BCA kit and adjusted for each group. Fifty microliters homogenate was further diluted with $300 \mu \mathrm{L} 30 \mathrm{mM}$ Tris-acetic acid containing $0.1 \%$ Triton $\mathrm{X}-100$ and $25 \mu \mathrm{Ci}$ of radioactive tyrosine $\mathrm{HCl}(0.4 \mathrm{mCi} /$ $\mathrm{nMol}$ of $\mathrm{L}-\left[\right.$ ring- $\left.3,5-{ }^{3} \mathrm{H}\right]$ tyrosine), $50 \mathrm{nMol}$ of the cofactor 6, 7-dimethyl 5, 6, 7, 8-tetrahydropterin $\left(\mathrm{DMPH}_{4}\right.$, Sigma, St. Louis, MO, USA), 5000 units of catalase, and $5 \mathrm{nM}$ DTT in $100 \mathrm{mM}$ potassium phosphate, $\mathrm{pH} 6.0$ for incubation at $37{ }^{\circ} \mathrm{C}$ for $20 \mathrm{~min}$. Then, aliquots were taken and placed in $5 \%(\mathrm{v} / \mathrm{v})$ perchloric acid to stop the reaction. Unreacted tyrosine and the product DOPA were absorbed with $1.0 \mathrm{~mL}$ of an aqueous slurry of activated charcoal (in $0.1 \mathrm{M} \mathrm{HCl}$ ) and the released $\left[{ }^{3} \mathrm{H}\right] \mathrm{H}_{2} \mathrm{O}$ was analyzed by liquid scintillation counting. TH utilizes $\mathrm{O}_{2}$ to produce DOPA, and $\left[{ }^{3} \mathrm{H}\right] \mathrm{H}_{2} \mathrm{O}$ is derived from $\left[{ }^{3} \mathrm{H}\right]$ tyrosine. The $\mathrm{TH}$ activity was expressed as radiation value of $\left[{ }^{3} \mathrm{H}\right] \mathrm{H}_{2} \mathrm{O}$ produced per group.

For the TH activity assay in yeast, yeast was cultured in SD media at $30{ }^{\circ} \mathrm{C}$ for $24 \mathrm{~h}$ to $\mathrm{OD}=1$. Yeast was collected by centrifugation at $3000 \mathrm{~g}$ for $5 \mathrm{~min}$ and then transferred to SGR media supplemented with metals or metal chelators, as indicated in each experiment; 500 $\mathrm{nMol} \mathrm{DMPH}_{4}$ (Sigma, St. Louis, MO, USA) and $10 \mu \mathrm{L}$ $\mathrm{L}$-[ring- $3,5-{ }^{3} \mathrm{H}$ ]tyrosine were added in the media and then the yeast was further cultured at $30{ }^{\circ} \mathrm{C}$ for $24 \mathrm{~h}$. Cells were removed after centrifugation at $12,000 \mathrm{~g}$ for $15 \mathrm{~min}$. The supernatant was transferred to a new container. Unreacted tyrosine and the product DOPA were absorbed with $1.0 \mathrm{~mL}$ of an aqueous slurry of activated charcoal (in $0.1 \mathrm{M} \mathrm{HCl}$ ) and the released $\left[{ }^{3} \mathrm{H}\right] \mathrm{H}_{2} \mathrm{O}$ was analyzed by liquid scintillation counting. The $\mathrm{TH}$ activity was expressed as radiation value of $\left[{ }^{3} \mathrm{H}\right] \mathrm{H}_{2} \mathrm{O}$ produced per group. All experiments were repeated at least three times.

\section{Statistical analysis}

All data were recorded and analyzed with Microsoft Excel and GraphPad Prism (version 6.00; La Jolla, CA, USA). Data were analyzed by Student's $t$-test between the groups, and ANOVA was used for multiple comparison. Statistical results were presented as means \pm SEM. Asterisks indicate critical levels of significance $\left(" p<0.05,{ }^{* * *} p<0.01,{ }^{* * * *} p<0.001\right)$.

\section{Abbreviations}

DA: Dopamine; PD: Parkinson's disease; TH: Tyrosine hydroxylase;

Catsup: Catecholamines up; CNS: Central nervous system; OE: Overexpression; ALP: Alkaline phosphatase; TPEN: N,N,N',N'-tetrakis(2-

pyridylmethyl)ethylenediamine; BPS: Bathophenanthrolinedisulfonic acid; BH4: Tetrahydrobiopterin; DMPH4: 6, 7-Dimethyl 5, 6, 7, 8-tetrahydropterin; GTPCH: GTP cyclohydrolase I; ICP-MS: Inductively coupled plasma mass spectrometry

\section{Supplementary Information}

The online version contains supplementary material available at https:/doi. org/10.1186/s12915-021-01168-0.

\begin{abstract}
Additional file 1: Figure S1. Quantification of the signal intensity in Figure 4B. No obvious and consistent changes were observed. All values are presented as mean \pm SEM and included in Additional file 2: table S13; $n=4$. Figure S2. L-dopa production is promoted by iron and suppressed by zinc in INVSCI. (A) The generation of L-dopa in INVSCI was elevated by iron and inhibited by zinc added in the iron deficient medium. All values are presented as mean \pm SEM and included in Additional file 2: table S14; $\mathrm{n}=3$. ${ }^{*} \mathrm{p}<0.05,{ }^{* *} \mathrm{p}<0.01$, ${ }^{* * *} \mathrm{p}<0.001$. (B) L-dopa production in INVSC1 was inhibited by Catsup expression in the iron abundant medium. All values are presented as mean \pm SEM and included in Additional file 2: table S15; $n=3 .{ }^{*} p<0.05$. Figure S3. $\mathrm{TH}$ protein levels were not much changed under metal treatments. A coomassie blue staining of protein gel was performed to test the effect of iron and zinc on the expression of TH in E. coli. Figure S4. ICP-MS indicated that zinc competes with iron in binding with Drosophila TH. Zinc binding sites could exceed 1 at high zinc concentrations, possibly due to additional non-specific bindings. All values presented in this graph are included in Additional file 2: table S12. Figure S5. Effects of modulating zinc and iron homeostasis on the mobility and survival of flies in the absence of rotenone. (A) The mobility of flies of various genotypes raised on normal food. $n=6 .{ }^{* *} p<0.001$. (B) The lifespan of flies of various genotypes in the absence of rotenone. Genotypes in (A) and (B) of the flies used were TH-Gal4>W ${ }^{1118}$ (control), THGal4>Catsup RNAi, TH-Gal4>Catsup OE, TH-Gal4>dZnT7 OE, TH-Gal4>Mvl $O E$. All values are presented as mean $\pm S E M ; n=6$. Figure $\mathbf{S 6}$. $\mathrm{TH}$ phosphorylation in SH-SY5Y cells under different metal or metal chelator treatments. Gel blot was cut around the potential target region as indicated by the markers and then hybridized to $\mathrm{TH}$ and $\mathrm{P}-\mathrm{TH}$ respectively. These are the original gel exposure pictures. Related to Figure $4 \mathrm{~B}$.

Additional file 2. The individual raw data values of figures and Additional files for number of replicates $\leq 6$. All the data are cited in the figure legend.
\end{abstract}

\section{Acknowledgements}

Yeast strains were originally kind gifts from David Eide, PhD. Fly stocks were from the Bloomington Stock Center (Bloomington, IN, USA) and the Vienna Drosophila RNAi Center. We are thankful for the technical help from Junxuan $\mathrm{Xu}$, Zhiqing Wang, Xuekai Ma, Xiaowen $\mathrm{Ji}$, and other lab members. We also thank Zhiwu Zhu, PhD, and Miao Liu for reading and commenting on this manuscript.

\section{Authors' contributions}

GX contributed to the methodology, investigation, data curation, formal analysis, conceptualization, and writing —original draft and writing —review. $\mathrm{MZ}$ contributed to the methodology, investigation, data curation, formal analysis, conceptualization, and writing - review. ZL and FD contributed to the methodology and data curation. BZ contributed to the conceptualization, writing - review and editing, project administration, supervision, and funding acquisition. All authors read and approved the final manuscript.

\section{Funding}

This study was supported by the National Key Research and Development Program of China (2018YFA0900100) and the National Natural Science Foundation of China $(31971087,31671284)$. 


\section{Availability of data and materials}

All data generated or analyzed during this study are included in this published article and its supplementary information files.

\section{Declarations}

Ethics approval and consent to participate

Not applicable.

\section{Consent for publication}

Not applicable.

\section{Competing interests}

The authors declare that they have no competing interests.

\section{Author details}

${ }^{1}$ State Key Laboratory of Membrane Biology, School of Life Sciences, Tsinghua University, Beijing 100084, China. ${ }^{2}$ School of Food and Biological Engineering, Hefei University of Technology, Hefei 230009, Anhui, China.

Received: 12 March 2021 Accepted: 15 October 2021

Published online: 03 November 2021

\section{References}

1. Massano J, Bhatia KP. Clinical approach to Parkinson's disease: features, diagnosis, and principles of management. Cold Spring Harb Perspect Med. 2012;2(6):a008870. https://doi.org/10.1101/cshperspect.a008870.

2. Neckameyer WS. Multiple roles for dopamine in Drosophila development. Dev Biol. 1996;176(2):209-19. https://doi.org/10.1006/dbio.1996.0128.

3. Berridge CW, Devilbiss DM. Psychostimulants as cognitive enhancers: the prefrontal cortex, catecholamines, and attention-deficit/hyperactivity disorder. Biol Psychiatry. 2011;69(12):e101-11. https://doi.org/10.1016/j. biopsych.2010.06.023.

4. Haenisch B, Bonisch H. Depression and antidepressants: insights from knockout of dopamine, serotonin or noradrenaline re-uptake transporters. Pharmacol Ther. 2011;129(3):352-68. https://doi.org/10.1016/j.pharmthera.2 010.12.002.

5. Berke JD. What does dopamine mean? Nat Neurosci. 2018;21(6):787-93. https://doi.org/10.1038/s41593-018-0152-y.

6. Daubner SC, Le T, Wang S. Tyrosine hydroxylase and regulation of dopamine synthesis. Arch Biochem Biophys. 2011;508(1):1-12. https://doi. org/10.1016/j.abb.2010.12.017.

7. Tekin I, Roskoski R Jr, Carkaci-Salli N, Vrana KE. Complex molecular regulation of tyrosine hydroxylase. J Neural Transm (Vienna). 2014;121(12): 1451-81. https://doi.org/10.1007/s00702-014-1238-7.

8. Memmert S, Damanaki A, Nogueira AVB, Nokhbehsaim M, Götz W, Cirelli JA, et al. Regulation of tyrosine hydroxylase in periodontal fibroblasts and tissues by obesity-associated stimuli. Cell Tissue Res. 2019;375(3):619-28. https://doi.org/10.1007/s00441-018-2941-8.

9. Nagatsu T, Nagatsu I. Tyrosine hydroxylase $(T H)$, its cofactor tetrahydrobiopterin (BH4), other catecholamine-related enzymes, and their human genes in relation to the drug and gene therapies of Parkinson's disease (PD): historical overview and future prospects. J Neural Transm (Vienna). 2016;123(11):1255-78. https://doi.org/10.1007/s00702-016-1596-4.

10. Zhu Y, Zhang J, Zeng Y. Overview of tyrosine hydroxylase in Parkinson's disease. CNS Neurol Disord Drug Targets. 2012;11(4):350-8. https://doi.org/1 0.2174/187152712800792901.

11. Woimant F, Trocello JM. Disorders of heavy metals. Handb Clin Neurol. 2014;120:851-64. https://doi.org/10.1016/B978-0-7020-4087-0.00057-7.

12. Montgomery EB Jr. Heavy metals and the etiology of Parkinson's disease and other movement disorders. Toxicology. 1995;97(1-3):3-9. https://doi. org/10.1016/0300-483X(94)02962-T.

13. Sikk K, et al. Manganese-induced parkinsonism due to ephedrone abuse. Parkinsons Dis. 2011;2011:865319.

14. Singh N, Haldar S, Tripathi AK, McElwee MK, Horback K, Beserra A. Iron in neurodegenerative disorders of protein misfolding: a case of prion disorders and Parkinson's disease. Antioxid Redox Signal. 2014;21(3):471-84. https:// doi.org/10.1089/ars.2014.5874.

15. Dexter DT, Wells FR, Lee AJ, Agid F, Agid Y, Jenner P, et al. Increased nigral iron content and alterations in other metal ions occurring in brain in
Parkinson's disease. J Neurochem. 1989;52(6):1830-6. https://doi.org/1 0.1111/j.1471-4159.1989.tb07264.X.

16. Kumar V, Singh BK, Chauhan AK, Singh D, Patel DK, Singh C. Minocycline rescues from zinc-induced nigrostriatal dopaminergic neurodegeneration: biochemical and molecular interventions. Mol Neurobiol. 2016;53(5):276177. https://doi.org/10.1007/s12035-015-9137-y.

17. Kumar V, Singh D, Singh BK, Singh S, Mittra N, Jha RR, et al. Alpha-synuclein aggregation, ubiquitin proteasome system impairment, and L-Dopa response in zinc-induced parkinsonism: resemblance to sporadic Parkinson's disease. Mol Cell Biochem. 2018;444(1-2):149-60. https://doi.org/10.1007/s11 010-017-3239-y.

18. Dos Santos AB, et al. Hair in Parkinson's disease patients exhibits differences in calcium, iron and zinc concentrations measured by flame atomic absorption spectrometry - FAAS. J Trace Elem Med Biol. 2018;47:134-9. https://doi.org/10.1016/j.jtemb.2018.02.003.

19. Stathakis DG, Burton DY, Mclvor WE, Krishnakumar S, Wright TRF, O'Donnell $J M$. The catecholamines up (Catsup) protein of Drosophila melanogaster functions as a negative regulator of tyrosine hydroxylase activity. Genetics. 1999;153(1):361-82. https://doi.org/10.1093/genetics/153.1.361.

20. Chaudhuri A, Bowling K, Funderburk C, Lawal H, Inamdar A, Wang Z, et al. Interaction of genetic and environmental factors in a Drosophila parkinsonism model. J Neurosci. 2007;27(10):2457-67. https://doi.org/10.1 523/JNEUROSCI.4239-06.2007.

21. Wang Z, Ferdousy F, Lawal H, Huang Z, Daigle JG, Izevbaye I, et al. Catecholamines up integrates dopamine synthesis and synaptic trafficking. J Neurochem. 2011;119(6):1294-305. https://doi.org/10.1111/j.1471-4159.2011. 07517.x.

22. Hsouna A, Lawal HO, Izevbaye I, Hsu T, O'Donnell JM. Drosophila dopamine synthesis pathway genes regulate tracheal morphogenesis. Dev Biol. 2007; 308(1):30-43. https://doi.org/10.1016/j.ydbio.2007.04.047.

23. Harbison ST, Carbone MA, Ayroles JF, Stone EA, Lyman RF, Mackay TFC. Co-regulated transcriptional networks contribute to natural genetic variation in Drosophila sleep. Nat Genet. 2009;41(3):371-5. https://doi. org/10.1038/ng.330

24. Groth C, Sasamura T, Khanna MR, Whitley M, Fortini ME. Protein trafficking abnormalities in Drosophila tissues with impaired activity of the ZIP7 zinc transporter Catsup. Development. 2013;140(14):3018-27. https://doi.org/1 $0.1242 /$ dev. 088336

25. Begum NA, Kobayashi M, Moriwaki Y, Matsumoto M, Toyoshima K, Seya T. Mycobacterium bovis BCG cell wall and lipopolysaccharide induce a novel gene, BIGM103, encoding a 7-TM protein: identification of a new protein family having Zn-transporter and Zn-metalloprotease signatures. Genomics. 2002;80(6):630-45. https://doi.org/10.1006/geno.2002.7000.

26. Huang L, Kirschke CP, Zhang Y, Yu YY. The ZIP7 gene (SIc39a7) encodes a zinc transporter involved in zinc homeostasis of the Golgi apparatus. J Biol Chem. 2005;280(15):15456-63. https://doi.org/10.1074/jbc.M412188200.

27. Lee MG, Bin BH. Different Actions of Intracellular Zinc Transporters ZIP7 and ZIP13 Are Essential for Dermal Development. Int J Mol Sci. 2019;20(16). https://doi.org/10.3390/ijms20163941.

28. Tuncay E, Bitirim VC, Durak A, Carrat GRJ, Taylor KM, Rutter GA, et al. Hyperglycemia-induced changes in ZIP7 and ZnT7 expression cause Zn(2+) release from the sarco(endo)plasmic reticulum and mediate ER stress in the heart. Diabetes. 2017;66(5):1346-58. https://doi.org/10.2337/db16-1099.

29. Lye JC, Richards CD, Dechen K, Paterson D, de Jonge MD, Howard DL, et al. Systematic functional characterization of putative zinc transport genes and identification of zinc toxicosis phenotypes in Drosophila melanogaster. J Exp Biol. 2012;215(Pt 18):3254-65. https://doi.org/10.1242/jeb.069260.

30. Xiao G, Wan Z, Fan Q, Tang X, Zhou B. The metal transporter ZIP13 supplies iron into the secretory pathway in Drosophila melanogaster. Elife. 2014;3: e03191. https://doi.org/10.7554/eLife.03191.

31. Richards $C D$, Burke R. A fly's eye view of zinc homeostasis: novel insights into the genetic control of zinc metabolism from Drosophila. Arch Biochem Biophys. 2016;611:142-9. https://doi.org/10.1016/j.abb.2016.07.015.

32. Lye JC, Richards CD, Dechen K, Warr CG, Burke R. In vivo zinc toxicity phenotypes provide a sensitized background that suggests zinc transport activities for most of the Drosophila Zip and ZnT genes. J Biol Inorg Chem. 2013;18(3):323-32. https://doi.org/10.1007/s00775-013-0976-6.

33. Dechen K, Richards CD, Lye JC, Hwang JEC, Burke R. Compartmentalized zinc deficiency and toxicities caused by ZnT and Zip gene over expression result in specific phenotypes in Drosophila. Int J Biochem Cell Biol. 2015;60: 23-33. https://doi.org/10.1016/j.biocel.2014.12.017. 
34. Xiao G, Fan Q, Wang X, Zhou B. Huntington disease arises from a combinatory toxicity of polyglutamine and copper binding. Proc Natl Acad Sci U S A. 2013;110(37):14995-5000. https://doi.org/10.1073/pna S. 1308535110

35. Le Du MH, et al. Crystal structure of alkaline phosphatase from human placenta at $1.8 \mathrm{~A}$ resolution. Implication for a substrate specificity. J Biol Chem. 2001;276(12):9158-65. https://doi.org/10.1074/jbc.M009250200.

36. Suzuki T, Ishihara K, Migaki H, Matsuura W, Kohda A, Okumura K, et al. Zinc transporters, ZnT5 and ZnT7, are required for the activation of alkaline phosphatases, zinc-requiring enzymes that are glycosylphosphatidylinositolanchored to the cytoplasmic membrane. J Biol Chem. 2005;280(1):637-43. https://doi.org/10.1074/jbc.M411247200.

37. Zhao M, Zhou B. A distinctive sequence motif in the fourth transmembrane domain confers ZIP13 iron function in Drosophila melanogaster. Biochim Biophys Acta Mol Cell Res. 1867;2020(2):118607. https://doi.org/10.1016/j.bba mcr.2019.118607.

38. Friggi-Grelin F, Coulom H, Meller M, Gomez D, Hirsh J, Birman S. Targeted gene expression in Drosophila dopaminergic cells using regulatory sequences from tyrosine hydroxylase. J Neurobiol. 2003;54(4):618-27. https://doi.org/10.1002/neu.10185.

39. Wang $X$, Wu $Y$, Zhou B. Dietary zinc absorption is mediated by ZnT1 in Drosophila melanogaster. FASEB J. 2009;23(8):2650-61. https://doi.org/10.1 096/fj.08-126649.

40. Wang Z, Li X, Zhou B. Drosophila ZnT1 is essential in the intestine for dietary zinc absorption. Biochem Biophys Res Commun. 2020;533(4):100411. https://doi.org/10.1016/j.bbrc.2020.09.077.

41. Ohashi W, Kimura S, Iwanaga T, Furusawa $Y$, Irié $T$, Izumi H, et al. Zinc transporter SLC39A7/ZIP7 promotes intestinal epithelial self-renewal by resolving ER stress. PLoS Genet. 2016;12(10):e1006349. https://doi.org/10.13 71/journal.pgen.1006349.

42. Qin Q, Wang X, Zhou B. Functional studies of Drosophila zinc transporters reveal the mechanism for dietary zinc absorption and regulation. BMC Biol. 2013;11(1):101. https://doi.org/10.1186/1741-7007-11-101.

43. Dunkley PR, Bobrovskaya L, Graham ME, von Nagy-Felsobuki El, Dickson PW. Tyrosine hydroxylase phosphorylation: regulation and consequences. J Neurochem. 2004;91(5):1025-43. https://doi.org/10.1111/j.1471-4159.2004.02797.x.

44. Neckameyer WS, Quinn WG. Isolation and characterization of the gene for Drosophila tyrosine hydroxylase. Neuron. 1989;2(2):1167-75. https://doi. org/10.1016/0896-6273(89)90183-9.

45. Neckameyer WS, White K. Drosophila tyrosine hydroxylase is encoded by the pale locus. J Neurogenet. 1993;8(4):189-99. https://doi.org/10.3109/01 677069309083448

46. Neckameyer WS, Holt B, Paradowski TJ. Biochemical conservation of recombinant Drosophila tyrosine hydroxylase with its mammalian cognates. Biochem Genet. 2005;43(7-8):425-43. https://doi.org/10.1007/s10528-005-6781-3.

47. Vie A, et al. Differential regulation of Drosophila tyrosine hydroxylase isoforms by dopamine binding and CAMP-dependent phosphorylation. J Biol Chem. 1999;274(24):16788-95. https://doi.org/10.1074/jbc.274.24.16788.

48. Krishnakumar S, Burton D, Rasco J, Chen X, O'donnell J. Functional interactions between GTP cyclohydrolase I and tyrosine hydroxylase in Drosophila. J Neurogenet. 2000;14(1):1-23. https://doi.org/10.3109/01 677060009083474

49. O'Donnell JM, McLean JR, Reynolds ER. Molecular and developmental genetics of the Punch locus, a pterin biosynthesis gene in Drosophila melanogaster Dev Genet. 1989:10(3):273-86. https://doi.org/10.1002/dvg.1020100316.

50. Yamamoto K, Kobayashi N, Yoshitama K, Teramoto S, Komamine A. Isolation and purification of tyrosine hydroxylase from callus cultures of Portulaca grandiflora. Plant Cell Physiol. 2001;42(9):969-75. https:/doi.org/10.1093/pcp/pce125.

51. Kim DH, Shin M, Jung SH, Kim YJ, Jones WD. A fat-derived metabolite regulates a peptidergic feeding circuit in Drosophila. PLoS Biol. 2017;15(3): e2000532. https://doi.org/10.1371/journal.pbio.2000532.

52. Haavik J, et al. Recombinant human tyrosine hydroxylase isozymes. Reconstitution with iron and inhibitory effect of other metal ions. Eur J Biochem. 1991;199(2):371-8. https://doi.org/10.1111/j.1432-1033.1991.tb16133.x.

53. Haavik J, et al. The incorporation of divalent metal ions into recombinant human tyrosine hydroxylase apoenzymes studied by intrinsic fluorescence and 1H-NMR spectroscopy. Eur J Biochem. 1992;210(1):23-31. https://doi. org/10.1111/j.1432-1033.1992.tb17386.x.

54. Yuan M, Li X, Xiao J, Wang S. Molecular and functional analyses of COPT/ Ctr-type copper transporter-like gene family in rice. BMC Plant Biol. 2011; 11(1):69. https://doi.org/10.1186/1471-2229-11-69.
55. Qiao W, Ellis C, Steffen J, Wu CY, Eide DJ. Zinc status and vacuolar zinc transporters control alkaline phosphatase accumulation and activity in Saccharomyces cerevisiae. Mol Microbiol. 2009;72(2):320-34. https://doi. org/10.1111/j.1365-2958.2009.06644.x

56. Ramsey AJ, Daubner SC, Ehrlich Jl, Fitzpatrick PF. Identification of iron ligands in tyrosine hydroxylase by mutagenesis of conserved histidinyl residues. Protein Sci. 1995;4(10):2082-6. https://doi.org/10.1002/pro. 5560041013.

57. Braak H, Ghebremedhin E, Rüb U, Bratzke H, del Tredici K. Stages in the development of Parkinson's disease-related pathology. Cell Tissue Res. 2004; 318(1):121-34. https://doi.org/10.1007/s00441-004-0956-9.

58. Betarbet R, Sherer TB, MacKenzie G, Garcia-Osuna M, Panov AV, Greenamyre JT. Chronic systemic pesticide exposure reproduces features of Parkinson's disease. Nat Neurosci. 2000;3(12):1301-6. https://doi.org/10.1038/81834.

59. Sherer TB, Kim JH, Betarbet R, Greenamyre JT. Subcutaneous rotenone exposure causes highly selective dopaminergic degeneration and alphasynuclein aggregation. Exp Neurol. 2003;179(1):9-16. https://doi.org/10.1 006/exnr.2002.8072

60. Calap-Quintana P, González-Fernández J, Sebastiá-Ortega N, Llorens J, Moltó M. Drosophila melanogaster Models of Metal-Related Human Diseases and Metal Toxicity. Int J Mol Sci. 2017;18(7). https://doi.org/1 $0.3390 /$ ijms 18071456

61. Xiao G, Zhou B. ZIP13: a study of Drosophila offers an alternative explanation for the corresponding human disease. Front Genet. 2017;8:234.

62. Djuric A, Begic A, Gobeljic B, Stanojevic I, Ninkovic M, Vojvodic D, et al. Oxidative stress, bioelements and androgen status in testes of rats subacutely exposed to cadmium. Food Chem Toxicol. 2015;86:25-33. https://doi.org/10.1016/j.fct.2015.09.004.

63. Kuznetsova SS, Azarkina NV, Vygodina TV, Siletsky SA, Konstantinov AA. Zinc ions as cytochrome $C$ oxidase inhibitors: two sites of action. Biochemistry (Mosc). 2005;70(2):128-36. https://doi.org/10.1007/s10541-005-0091-6.

64. Maret W. The redox biology of redox-inert zinc ions. Free Radic Biol Med. 2019;134:311-26. https://doi.org/10.1016/j.freeradbiomed.2019.01.006.

65. Maret W. Zinc in Cellular Regulation: The Nature and Significance of "Zinc Signals". Int J Mol Sci. 2017;18(11). https://doi.org/10.3390/ijms18112285.

66. Ramsey AJ, Hillas PJ, Fitzpatrick PF. Characterization of the active site iron in tyrosine hydroxylase. Redox states of the iron. J Biol Chem. 1996;271(40): 24395-400. https://doi.org/10.1074/jbc.271.40.24395.

67. Bjorklund G, Stejskal V, Urbina MA, Dadar M, Chirumbolo S, Mutter J. Metals and Parkinson's disease: mechanisms and biochemical processes. Curr Med Chem. 2018;25(19):2198-214. https://doi.org/10.2174/092986732566617112 9124616.

68. Tosato M, Di Marco V. Metal Chelation Therapy and Parkinson's Disease: A Critical Review on the Thermodynamics of Complex Formation between Relevant Metal lons and Promising or Established Drugs. Biomolecules. 2019;9(7). https://doi.org/10.3390/biom9070269.

69. Thingholm TE, Ronnstrand L, Rosenberg PA. Why and how to investigate the role of protein phosphorylation in ZIP and ZnT zinc transporter activity and regulation. Cell Mol Life Sci. 2020;77(16):3085-102. https://doi.org/10.1 007/s00018-020-03473-3.

70. Chatterjee S, Sang TK, Lawless GM, Jackson GR. Dissociation of tau toxicity and phosphorylation: role of GSK-3beta, MARK and Cdk5 in a Drosophila model. Hum Mol Genet. 2009;18(1):164-77. https://doi.org/10.1093/hmg/ddn326.

71. Bergerman J, Blethen S. Determination of alkaline phosphatase isoenzymes. Clin Chim Acta. 1972;36(2):389-96. https://doi.org/10.1016/0009-8981(72 )90013-7.

72. EarleArnow L. Colorimetric determination of the components of 3,4 dihydroxyphenylalanine-tyrosine mixtures. J Biol Chem. 1937;118(2):531-7. https://doi.org/10.1016/S0021-9258(18)74509-2.

73. Rodriguez-Gomez JA, et al. Increased activity and expression of tyrosine hydroxylase in the rat substantia nigra after chronic treatment with nomifensine. Mol Pharmacol. 1997;52(4):641-7. https://doi.org/10.1124/mol. 52.4.641.

74. Borelli Ml, Rubio M, García ME, Flores LE, Gagliardino JJ. Tyrosine hydroxylase activity in the endocrine pancreas: changes induced by shortterm dietary manipulation. BMC Endocr Disord. 2003;3(1):2. https://doi.org/1 $0.1186 / 1472-6823-3-2$.

\section{Publisher's Note}

Springer Nature remains neutral with regard to jurisdictional claims in published maps and institutional affiliations. 